\title{
Distributed and On-Line Routing on Tori ${ }^{1}$
}

\author{
Tzuoo-Hawn Yeh, ${ }^{2}$ Cheng-Ming Kuo, ${ }^{2}$ Chin-Laung Lei, ${ }^{2}$ and Hsu-Chun Yen ${ }^{2}$
}

\begin{abstract}
In this paper we study the problem of assigning paths to packets on $N \times N$ tori in an on-line and distributed fashion. By on-line we mean that the routing decisions must be made without any knowledge of future requests. Being distributed is an equally important feature of our design, for such algorithms need not know the global configuration of the network in the process of routing packets. We use the technique of competitive analysis to measure the performance of our design. In addition to showing an $\Omega(\log N)$ lower bound on the competitive ratio, we present both deterministic and randomized algorithms which are $O(\log N)$ competitive with respect to the maximum load (i.e., congestion) on communication links.
\end{abstract}

Key Words. Routing, On-line algorithm, Competitive analysis, Distributed algorithm, Randomized algorithm.

1. Introduction. We study the routing problem, i.e., the problem of assigning paths to packets, in an on-line and distributed setting on tori. The routing problem is important in, among others, the packet delivering problem (see [14] and references therein), which is moving packets toward their destinations under the constraint that at most one packet is allowed to move along an edge in a time step. The goal of the delivering problem is to minimize the work-span, i.e., the number of time steps required to deliver all packets. To simplify the study of the packet delivering problem, a useful approach is to divide the problem into two subproblems, namely, the routing problem (studied in our paper and in, e.g., [15] and [20]) and the scheduling problem (studied in, e.g., [9], [11]-[13], [16], and [17]). The former concerns itself with assigning paths to packets, whereas the latter deals with deciding the order in which the packets along the predefined paths are moved in order to resolve conflicts if multiple packets are waiting for the same edge. Clearly, the effectiveness of an algorithm depends on the parameter chosen to measure the performance. The congestion and dilation measures are widely recognized as two of the most important objective functions in the study of the aforementioned problems. The congestion of a network is the maximum load on a link of the network, and the dilation measure refers to the length of the longest path among all the assigned paths in the network. The routing problem aims at reducing the congestion as well as the dilation, and the scheduling problem's goal is to minimize the work-span (given a set of paths of congestion and dilation).

In this paper we investigate the routing problem subject to the shortest-path constraint on $N \times N$ tori in an on-line and distributed setting with respect to the congestion measure. In an on-line manner, the assignment of a path to a packet has to be carried out without any knowledge of future requests. In the framework of distributed routing,

\footnotetext{
${ }^{1}$ A preliminary version of this paper was presented at the 8th International Symposium on Algorithms and Computation, ISAAC '97, under the title "Competitive Source Routing on Tori and Meshes."

${ }^{2}$ Department of Electrical Engineering, National Taiwan University, Taipei, Taiwan, Republic of China.
}

Received December 2, 1999; revised June 15, 2000. Communicated by M.-Y. Kao.

Online publication December 17, 2001. 
a node has no knowledge about the status of other nodes in the network. (In practice, distributed algorithms are easier to implement than their centralized counterparts.) Since the path associated with a packet must be determined in the routing phase and before the scheduling phase is started, the path is to be determined on its source node. In our setting, the path of a packet whose source node is $s$ depends only on those packets whose source nodes are $s$ as well. Like many existing routing algorithms and implementations which are based upon the shortest path assumption (see the survey paper by Leighton [14] and, for example, [4]), we restrict ourselves to routing algorithms which operate in the shortest path mode (i.e., only paths of shortest length are eligible for assignment). Enforcing the shortest path constraint to routing ensures that the dilation and the total load in the network are minimized in the first place.

Competitive analysis [19] is used for analyzing the performance of our routing algorithms. An on-line algorithm is said to be $\alpha$ competitive, or has competitive ratio $\alpha$, if, for any request sequence, (cost of the on-line algorithm) $\leq \alpha \times$ (cost of the optimal off-line algorithm $)+$ (some additive term), in which the additive term is independent of the request sequence. The competitive ratio reports the loss in performance due to on-line decision making. The notion of competitiveness for on-line algorithms can be applied to comparing the performance of algorithms in a distributed setting, where a distributed algorithm that makes decisions locally is compared with an adversary that makes decisions with global views. Competitive analysis of problems in a distributed environment can be found in [1]-[3] and [6].

The main contributions of this paper include the following:

- The competitive ratio of any distributed algorithm (deterministic or randomized) is shown to be $\Omega(\log N)$. We assume oblivious adversaries (the weakest among the three classified in [8]) against randomized algorithms.

- We provide an $O(\log N)$ competitive deterministic algorithm. Given many packets from node $s$ to node $t$, the heuristic is to distribute, based upon the source node $s$ 's local view of the network, the load among all the intermediate nodes and edges evenly. The algorithm keeps some local estimates of the edge loads on each node and assigns paths based on this information. We use a simple and easy to compute strategy (although its correctness proof is rather involved) to make the heuristic on-line. That is, the strategy attempts to maintain even loads for each incoming packet.

- We devise an $O(\log N)$ competitive randomized algorithm. This algorithm is based on the same heuristic for the deterministic one. Using the technique of randomization, the space required by our deterministic algorithm can be entirely eliminated, yielding a memoryless randomized algorithm.

The additive terms in the competitive analysis of the two aforementioned algorithms are $O\left(N^{5}\right)$ and $O(N)$, respectively. This means that the algorithms provided in our paper may have poor performance on short input sequences. However, since routing algorithms are generally used continuously and repeatedly in a distributed system, the considered input sequence should be long and thus the large additive terms are irrelevant here. Therefore we place little effort in improving the large additive terms. Another point that is worth mentioning is that the shortest path constraint applies to the adversaries too in our analysis. We think this is a reasonable constraint for the adversaries because the main issue in our paper is to report the performance loss due to the distributed and 
on-line nature of routing algorithms versus adversaries with global knowledge about the network and the future. If the shortest path constraint is not placed on the adversaries, we think the adversaries are too powerful for us to provide an insight into our problem.

In what follows we compare and contrast our results with some of the related work in the literature. In [15] Maggs et al. studied the data management problem of placing and accessing shared objects that are read and written from the nodes in tree-connected networks, meshes, and clustered networks. As a special case, they provided distributed routing algorithms on $N \times N$ meshes that are $O(\log N)$ competitive, and they also showed the lower bound to be $\Omega(\log N)$. Unlike the shortest-path model assumed in our work, in [15] packets are allowed to travel along arbitrary (i.e., not necessarily shortest) paths in the network. They do not care about the length at all. In the worst case a path could be $\Omega(N)$ times longer than the shortest one (and by the technique they use, they are always of length $O(N)$ ). However, their (randomized) algorithm is strictly $O(\log N)$ competitive in that the additive term is zero for their algorithm, in contrast to the algorithms presented in our paper. The disparity of arbitrary and shortest paths constitutes the fundamental difference between their model and ours. To cope with the shortest-path requirement, we employ a new technique (which is based upon a locally optimal heuristic, and is completely different from that used in [15]) which, we feel, might have applications to the analysis of other routing related problems.

To a certain extent, our work is also related to the problem of virtual circuit routing in ATM networks (see, e.g., [18]). In this problem the goal is to construct paths between the specified pairs of endpoints involved in the communication, and, at the same time, reserve the required bandwidth for each such path. A nice survey on the competitive algorithms for the virtual circuit routing problem can be found in [18]. It is worth pointing out that the algorithms discussed in [18] are basically centralized. The distributed nature of our routing scheme differentiates our work from [18], even though our model can be formulated as a special case of the permanent virtual circuit routing problem in the cost minimization model where all requests require unit bandwidth and all edges have equal capacity.

Another problem that is relevant to our work is the path coloring problem in WDM (wavelength division multiplexing) optical networks. In this problem one has to find a path and a color for a request so that no two requests of the same color cross the same edge. The colors correspond to wavelengths in optic fiber links. The goal is to minimize the number of colors used. Bartal et al. [5] provided lower bounds for path coloring and many other related problems. On-line algorithms in the cost model have been studied by Bartal and Leonardi [7]. In particular, they provided an $O(\log N)$ competitive deterministic on-line algorithm (which is not distributed) as well as a matching $\Omega(\log N)$ lower bound for this problem on $N$-node meshes. As the congestion in the routing problem is a lower bound for the number of colors used in the path coloring problem, the $\Omega(\log N)$ lower bound derived in our work (for the routing problem) offers a lower bound for the path coloring problem in a distributed and on-line setting on $N \times N$ tori.

In [20] Srinivasan and Teo give a constant-factor approximation algorithm for the routing problem on arbitrary networks. Given a set of $m$ packets, the centralized algorithm selects paths for the packets that approximate the minimum value of $c+d$, where $c$ is the congestion and $d$ is the dilation, within a constant factor. In [12] Leighton et al. showed that given a set of packets with predefined paths, there exists a schedule for moving the 
packets to their destinations in $O(c+d)$ time steps using constant-size queues. The proof in [12], however, is not constructive. In [13] Leighton et al. give a centralized algorithm to find such a schedule. Combining the result in [20] for the routing problem and the result in [13] for the scheduling problem, one gets a centralized solution for the packet delivering problem. The results in our paper is an attempt to devise distributed algorithms for the routing problem. Attempts that aims at providing distributed solutions for the scheduling problem can be found in [16] and [17].

The remainder of this paper is organized as follows. The basic notations and definitions are given in Section 2. In Section 3 the lower bound $\Omega(\log N)$ on the competitive ratio of the routing problem is derived. In Section 4 we give a deterministic algorithm and prove that its competitive ratio is $O(\log N)$. An $O(\log N)$ competitive randomized algorithm (which is memoryless) is established in Section 5.

2. Preliminaries. An $N \times N$ torus is a directed graph $G=(V, E)$. The nodes are denoted by integer pairs $(x, y)$, where $0 \leq x, y<N$. Node $(0,0)$ is at the upper-left corner. For each node $v=(x, y) \in V$, there are four out-going edges connecting to the following four nodes: $(x,(y+1) \bmod N),(x,(y-1) \bmod N),((x+1) \bmod N, y)$, and $((x-1) \bmod N, y)$. An edge from node $u$ to node $v$ is denoted as $(u, v)$.

A path $\tau$ of length $l$ from node $s$ to node $t$ is a sequence of $l$ connected edges $e_{1} e_{2} \cdots e_{l}$, such that $e_{i}=\left(u_{i}, u_{i+1}\right), 1 \leq i \leq l, s=u_{1}$, and $t=u_{l+1}$, for some nodes $u_{1}, u_{2}, \ldots$, $u_{l+1}$. The edge $e_{i}$ and the node $u_{i}, 1 \leq i \leq l$, are the $i$ th edge and the $i$ th node on $\tau$, respectively, and the node $t=u_{l+1}$ is the $(l+1)$ th node on $\tau$. A path $\tau$ from node $s$ to node $t$ is a shortest path if its length is no more than the length of any other path from $s$ to $t$. In this paper we use the terms "shortest path" and "minimum path" interchangeably. The distance between $s$ and $t$ is the length of a shortest path from $s$ to $t$.

A packet $\delta$ from node $s$ to node $t$ is denoted by the pair $\langle\langle s, t\rangle\rangle$. Nodes $s$ and $t$ are the source and target nodes, respectively. An input $I$ of length $m$ is a sequence of $m$ packets $\delta_{1} \delta_{2} \cdots \delta_{m}$. Define $(I)_{k}, 1 \leq k \leq m$, to be the sequence $\delta_{1} \delta_{2} \cdots \delta_{k}$. Define $\langle I\rangle_{v}, v \in V$, to be the subsequence of $I$ that comprises of all the packets in $I$ whose source nodes are $v$.

The on-line source routing problem considered in this paper is as follows. Given an input sequence $I$, find a minimum path $\tau_{\delta}$ for each packet $\delta=\langle\langle s, t\rangle\rangle \in I$ under the constraint that $\tau_{\delta}$ depends on $\left\langle(I)_{k}\right\rangle_{s}$ only, where $k$ is the position of the packet $\delta$ in the sequence $I$. Given an algorithm $\mathrm{A}$ and an input $I$, the load on edge $e \in E$, denoted as $\operatorname{load}_{\mathrm{A}}(I, e)$, is the number of packets whose paths contain $e$. Similarly, define $\operatorname{load}_{\mathrm{A}}(I, v)$, the load on node $v \in V$, to be the number of packets in $I$ whose paths contain $v$. The cost (or the congestion) of algorithm A on input $I$, denoted as $\operatorname{cost}_{\mathrm{A}}(I)$, is defined as $\max _{e \in E}\left(\operatorname{load}_{\mathrm{A}}(I, e)\right)$.

An algorithm $\mathrm{A}$ is $\alpha_{\mathrm{A}}$ competitive if there exists an additive term $\beta$ such that, for any input $I$,

$$
\operatorname{cost}_{\mathrm{A}}(I) \leq \alpha_{\mathrm{A}} \times \operatorname{cost}_{\mathrm{ADV}}(I)+\beta,
$$

where the additive term is independent of $I$. Here the adversary is abbreviated as ADV. If $A$ is randomized, the cost is defined as an expectation. The inequality becomes

$$
\exp \left(\operatorname{cost}_{\mathrm{A}}(I)\right) \leq \alpha_{\mathrm{A}} \times \operatorname{cost}_{\mathrm{ADV}}(I)+\beta .
$$

For randomized algorithms we assume an oblivious adversary [8]. 
Two paths $\tau_{1}$ and $\tau_{2}$ are edge-disjoint if $\tau_{1}$ and $\tau_{2}$ do not share any edge. A set of paths is edge-disjoint if any two paths in the set are edge-disjoint. We say that the pair of integers $\left[\left[x_{3}, y_{3}\right]\right]$ is the distance vector from node $s=\left(x_{1}, y_{1}\right)$ to node $t=\left(x_{2}, y_{2}\right)$ (or the distance vector of a packet $\langle\langle s, t\rangle\rangle)$ if $\left(x_{1}+x_{3}\right) \bmod N=x_{2},\left(y_{1}+y_{3}\right) \bmod N=y_{2}$, and $\left|x_{3}\right|+\left|y_{3}\right|$ equals the distance from $s$ to $t$. When $N$ is odd, the distance vector from $s$ to $t$ is unique. However, this is not the case when $N$ is even. As an example, consider the $6 \times 6$ torus. [[3, 3]], [[-3,3]], [[3, -3]], and [[-3, -3]] are distance vectors from node $(0,0)$ to node $(3,3)$. This and related irregularities are inconvenient in the analysis and presentation of this problem, so we assume in this paper that $N$ is odd. The even case only induces a constant factor to the upper and lower bounds. Since the orders of the bounds we will derive are logarithmic in $N$, the constant factor is irrelevant.

Please note that in this paper we adopt the following convention to make our notations cleaner. We use the letters $s, t, u$, and $v$ to denote nodes; the letter $e$ to denote edges; the Greek letter $\delta$ to denote a packet; the capital letter $I$ to denote an input; and the letters $i$, $j, x$, and $y$ to denote integers. To distinguish nodes from distance vectors (both of which are formed by a pair of integers), nodes are denoted by integer pairs in parentheses such as $(x, y)$, while distance vectors are denoted by integer pairs in double square brackets such as [[x,y]]. Similarly, to distinguish edges from packets (both of which are formed by a pair of nodes), edges are denoted by node pairs in parentheses such as $(u, v)$, while packets are denoted by node pairs in double angle brackets such as $\langle\langle s, t\rangle\rangle$. The notation $\langle i, j\rangle$ is left for a construction called a slice node in Section 4.

Consider a packet $\delta=\langle\langle s, t\rangle\rangle$. Define the $i$ th slice of edges for $\delta$, denoted as $S E_{\delta}(i)$, to be the set of edges $\{e \mid e$ is the $i$ th edge on a shortest path from $s$ to $t$. $\}$ Similarly, we define the $i$ th slice of nodes for $\delta$, denoted as $S V_{\delta}(i)$, to be the set of nodes $\{v \mid v$ is the $i$ th node on a shortest path from $s$ to $t$. $\}$ See Figure 1 for an example. For convenience, we use $s+[[x, y]]$, where $s=\left(x_{s}, y_{s}\right)$ is a node and $[[x, y]]$ is a distance vector, to denote the node $\left(\left(x_{s}+x\right) \bmod N,\left(y_{s}+y\right) \bmod N\right)$.

3. The Problem Lower Bound. In this section we show that the competitive ratio for any on-line source routing algorithm on $N \times N$ tori is $\Omega(\log N)$. This lower bound applies to both deterministic and randomized algorithms. For randomized algorithms, the costs are taken to be the expected costs and the adversary is assumed to be oblivious. Note that this lower bound applies to shortest path algorithms only and the adversaries are also restricted by the shortest path constraint.

THEOREM 1. For any on-line source routing algorithm $\mathrm{A}$ on $N \times N$ tori, the competitive ratio of $\mathrm{A}$ is $\Omega(\log N)$.

The proof proceeds as follows: For any integer $m$ and any edge $e \in E$, we construct an input $I_{e}$ such that

CLAIM 1. $\max _{e \in E} \operatorname{load}_{\mathrm{A}}\left(I_{e}, e\right)=\Omega(m \log N)$, which implies that $\operatorname{cost}_{\mathrm{A}}\left(I_{e}\right)=\Omega(m \log N)$.

CLAIM 2. $\operatorname{cost}_{\mathrm{ADV}}\left(I_{e}\right)=O(m)$ for any $I_{e}$. 


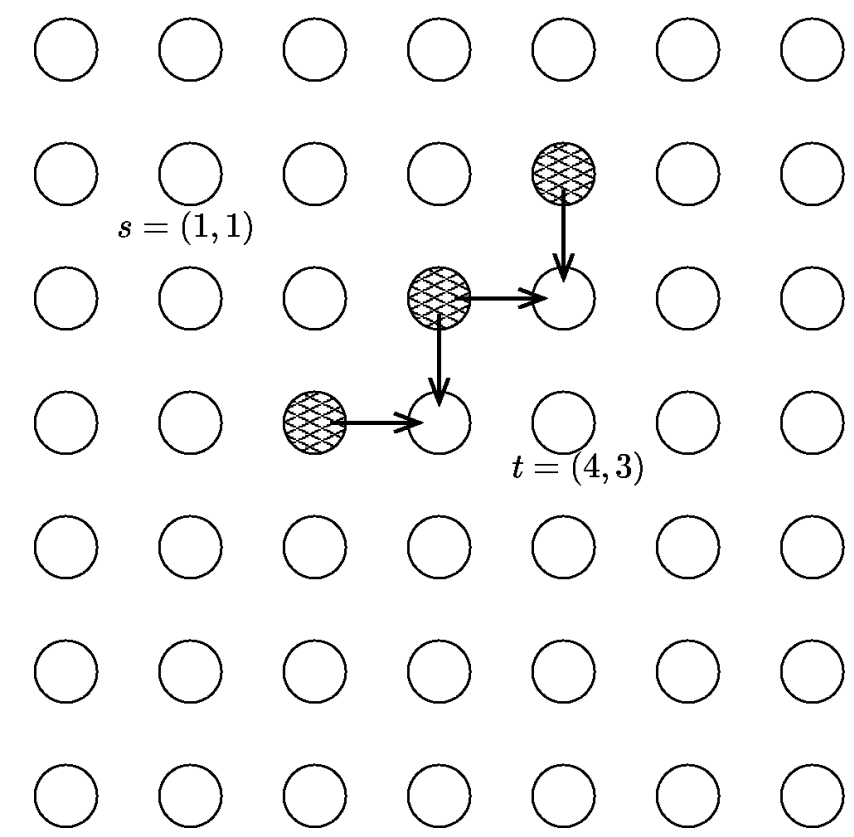

Fig. 1. $S V_{\delta}(4)$ and $S E_{\delta}(4)$ in a $7 \times 7$ torus. (The shaded nodes and the indicated edges.)

Before proving Claims 1 and 2, we need to give some additional terminologies. Two packets $\left\langle\left\langle s_{1}, t_{1}\right\rangle\right\rangle$ and $\left\langle\left\langle s_{2}, t_{2}\right\rangle\right\rangle$, where $s_{1}, s_{2}, t_{1}$, and $t_{2}$ are nodes, are equivalent if $s_{1}=s_{2}$ and $t_{1}=t_{2}$. Let $e=(u, v)$ be an edge and let $\left[\left[x_{e}, y_{e}\right]\right]$ be the distance vector from $u$ to $v$. Let $\delta=\langle\langle s, t\rangle\rangle$ be a packet and let $\left[\left[x_{\delta}, y_{\delta}\right]\right]$ be the distance vector of $\delta$. We say that $\delta$ is a left (respectively, right) packet to $e$ if $x_{\delta} y_{e}+y_{\delta} x_{e} \geq 0$ (respectively, $x_{\delta} y_{e}+y_{\delta} x_{e}<0$ ). Let $r=(N-1) / 2$. An input $I$ is a canonical input if $I$ is a sequence of $m$ equivalent packets whose distance vectors are $[[x, y]]$, where $|x|=|y|=r$. Note that there are $4 N^{2}$ distinct canonical inputs because each node in $V$ is the source of four canonical inputs. Since the packets in a canonical input $I$ are equivalent, without ambiguity, we define $S E_{I}(i)=S E_{\delta}(i)$ and $S V_{I}(i)=S V_{\delta}(i)$, where $\delta$ is a packet in $I$. Note that $\left|S E_{I}(i)\right|=2 i$, $1 \leq i \leq r$, and $\left|S V_{I}(i)\right|=i, 1 \leq i \leq r+1$.

Define the left canonical set $L C S_{e}(i), 1 \leq i \leq r$, to be the set of canonical inputs $\left\{I \mid I\right.$ is a canonical input, packets in $I$ are left to $e$, and $\left.e \in S E_{I}(i)\right\}$. See Figure 2 for an example. Symmetrically, define the right canonical set $R C S_{e}(i)=\{I \mid I$ is a canonical input, packets in $I$ are right to $e$, and $\left.e \in S E_{I}(i)\right\}$, for $1 \leq i \leq r$. Note that $\left|L C S_{e}(i)\right|=\left|R C S_{e}(i)\right|=i$. Next, we define $L_{e}(i)$ to be the input in $L C S_{e}(i)$ that causes the maximum load on $e$. Symmetrically, define $R_{e}(i)$ to be the input in $R C S_{e}(i)$ that causes the maximum load on $e$. Define $L I_{e}=\bigcup_{i=1}^{r} L_{e}(i)$ and $R I_{e}=\bigcup_{i=1}^{r} R_{e}(i)$. Finally, let $I_{e}=L I_{e}$ if $\operatorname{load}_{\mathrm{A}}\left(L I_{e}, e\right) \geq \operatorname{load}_{\mathrm{A}}\left(R I_{e}, e\right)$, otherwise, let $I_{e}=R I_{e}$. Note that $I_{e}, L I_{e}, L_{e}(i)$ depend on the given algorithm while $L C S_{e}(i)$ and $S E_{I}(i)$ do not.

Claims 1 and 2 are proved in Lemmas 1 and 2, respectively. 


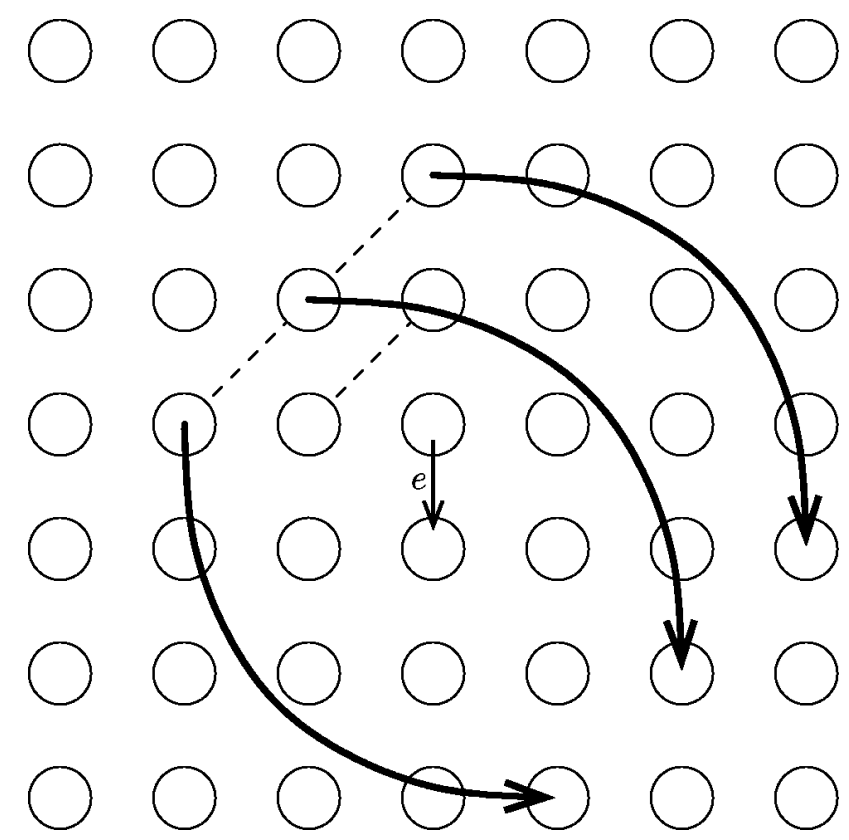

Fig. 2. $L C S_{e}(3)$ in a $7 \times 7$ torus.

LEMMA 1. Given an algorithm $\mathrm{A}, \max _{e \in E} \operatorname{load}_{\mathrm{A}}\left(I_{e}, e\right)=\Omega(m \log N)$.

ProOF. Let $\bar{e}$ be the edge that $\operatorname{load}_{\mathrm{A}}\left(I_{\bar{e}}, \bar{e}\right)$ has maximized. Since $|E|=4 N^{2}$,

$$
\operatorname{load}_{\mathrm{A}}\left(I_{\bar{e}}, \bar{e}\right) \geq \frac{1}{4 N^{2}} \sum_{e \in E} \operatorname{load}_{\mathrm{A}}\left(I_{e}, e\right)
$$

By the definition of $I_{e}$,

$$
\frac{1}{4 N^{2}} \sum_{e \in E} \operatorname{load}_{\mathrm{A}}\left(I_{e}, e\right) \geq \frac{1}{2} \cdot \frac{1}{4 N^{2}} \sum_{e \in E}\left(\operatorname{load}_{\mathrm{A}}\left(L I_{e}, e\right)+\operatorname{load}_{\mathrm{A}}\left(R I_{e}, e\right)\right)
$$

By the definition of $L I_{e}$ and the fact that $\mathrm{A}$ is a source routing algorithm, any two inputs $L_{e}\left(i_{1}\right)$ and $L_{e}\left(i_{2}\right)$ in $L I_{e}, i_{1} \neq i_{2}$, are processed independently by A. $\operatorname{So}_{\operatorname{load}_{\mathrm{A}}}\left(L I_{e}, e\right)=$ $\sum_{i=1}^{r} \operatorname{load}_{\mathrm{A}}\left(L_{e}(i), e\right)$. Therefore,

$$
\begin{aligned}
& \frac{1}{2} \cdot \frac{1}{4 N^{2}} \sum_{e \in E}\left(\operatorname{load}_{\mathrm{A}}\left(L I_{e}, e\right)+\operatorname{load}_{\mathrm{A}}\left(R I_{e}, e\right)\right) \\
& =\frac{1}{8 N^{2}} \sum_{e \in E} \sum_{i=1}^{r}\left(\operatorname{load}_{\mathrm{A}}\left(L_{e}(i), e\right)+\operatorname{load}_{\mathrm{A}}\left(R_{e}(i), e\right)\right) .
\end{aligned}
$$


By $L_{e}(i)$ 's definition,

$$
\begin{aligned}
\frac{1}{8 N^{2}} \sum_{e \in E} \sum_{i=1}^{r} & \left(\operatorname{load}_{\mathrm{A}}\left(L_{e}(i), e\right)+\operatorname{load}_{\mathrm{A}}\left(R_{e}(i), e\right)\right) \\
& \geq \frac{1}{8 N^{2}} \sum_{i=1}^{r}\left(\frac{1}{i} \sum_{e \in E}\left(\sum_{I \in\left(L C S_{e}(i) \cup R C S_{e}(i)\right)} \operatorname{load}_{\mathrm{A}}(I, e)\right)\right) .
\end{aligned}
$$

Looking closely at the term $\sum_{e \in E}\left(\sum_{I \in\left(L C S_{e}(i) \cup R C S_{e}(i)\right)} \operatorname{load}_{\mathrm{A}}(I, e)\right)$, we get

$$
\sum_{e \in E}\left(\sum_{I \in\left(L C S_{e}(i) \cup R C S_{e}(i)\right)} \operatorname{load}_{\mathrm{A}}(I, e)\right)=\sum_{I \text { is a canonical input }}\left(\sum_{e \in S E_{I}(i)} \operatorname{load}_{\mathrm{A}}(I, e)\right) .
$$

Because $\sum_{e \in S E_{I}(i)} \operatorname{load}_{\mathrm{A}}(I, e)=m$ and there are $4 N^{2}$ distinct canonical inputs,

$$
\sum_{I \text { is a canonical input }}\left(\sum_{e \in S E_{I}(i)} \operatorname{load}_{\mathrm{A}}(I, e)\right)=4 N^{2} \cdot m
$$

Therefore,

$$
\begin{aligned}
\operatorname{load}_{\mathrm{A}}\left(I_{\bar{e}}, \bar{e}\right) & \geq \frac{1}{8 N^{2}} \sum_{i=1}^{r}\left(\frac{1}{i} \cdot 4 N^{2} \cdot m\right) \\
& =\frac{m}{2} \cdot \sum_{i=1}^{r} \frac{1}{i} \geq \frac{m}{2} \ln r \\
& =\Omega(m \log N) . \quad\left(\text { Recall that } r=\frac{N-1}{2} .\right)
\end{aligned}
$$

LEMMA 2. $\operatorname{cost}_{\mathrm{ADV}}\left(I_{e}\right)=O(m)$ for any $I_{e}$.

PROOF. Because all edges in a torus are symmetric, without loss of generality, let $e$ be an edge from some node $v$ to node $v+[[0,1]]$. Because the $+x$ and $-x$ directions are symmetric in a torus, without loss of generality, we assume $I_{e}=L I_{e}$. By the definitions, $L I_{e}=\bigcup_{i=1}^{r} L_{e}(i)$, and $L_{e}(i)$ is one of the canonical inputs in $L C S_{e}(i)$, depending on what on-line source routing algorithm $A$ we are dealing with is. So we have to show that the lemma holds when $L_{e}(i)$ is any one of the inputs in ${ } C S_{e}(i)$.

Let node $S_{i}=v+[[0,-i]]$ and node $S_{-i}=v+[[-i, 0]]$, for $0 \leq i \leq r-1$. Let node $T_{i},-(r-1) \leq i \leq r-1$, be $S_{i}+[[r, r]]$. Assume that the packets in $L_{e}(i)$ are from node $s_{i}$ to node $t_{i}=s_{i}+[[r, r]], 1 \leq i \leq r$. Note that $s_{i}$ and $t_{i}$ are on the lines $\overline{S_{i-1} S_{-(i-1)}}$ and $\overline{T_{i-1} T_{-(i-1)}}$, respectively. Here we use $\overline{A B}$ to denote the set of nodes that are on an imaginary line from node $A$ to node $B$, including the endpoint nodes $A$ and $B$. We show in the following how to construct a minimum path connecting $s_{i}$ and $t_{i}$ for each $i, 1 \leq i \leq r$, such that the paths are edge-disjoint. The adversary can pump the $m$ equivalent packets $\left\langle\left\langle s_{i}, t_{i}\right\rangle\right\rangle$ in each $L_{e}(i)$ through the minimum path. It then follows that $\operatorname{cost}_{\mathrm{ADV}}\left(I_{e}\right)=m$. The construction of the paths is divided into the following three 


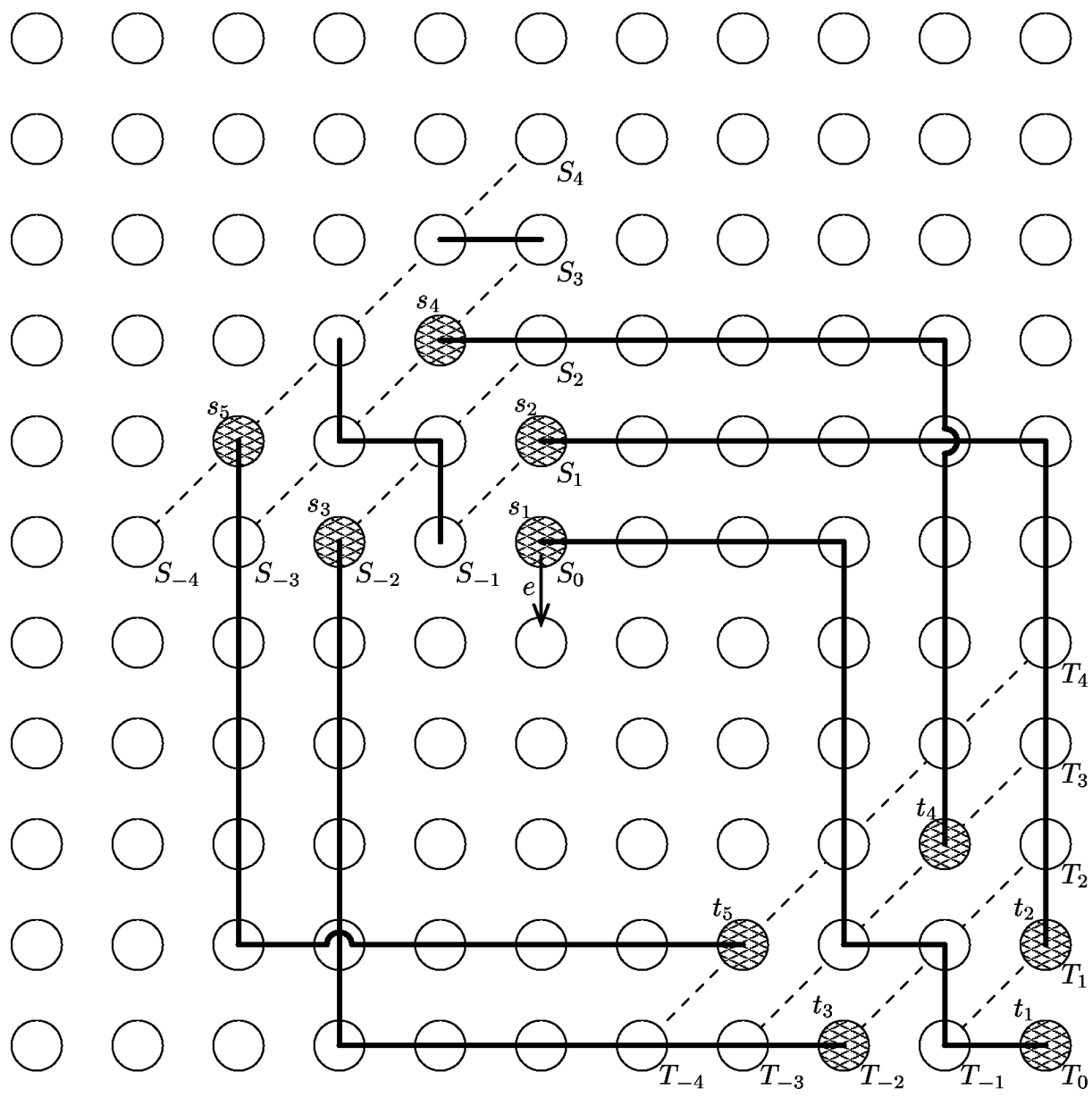

Fig. 3. The paths constructed by the adversary.

parts, each of which deals with a subsection of the paths. They can be connected to form the resulting paths. See Figure 3 for an example. In this figure the paths in the triangle $S_{0} S_{4} S_{-4}$ are constructed in P1, the paths in $T_{0} T_{4} T_{-4}$ are constructed in P2, and the remaining paths are constructed in $\mathrm{P} 3$. The paths connected to $S_{3}$ and $S_{-1}$ are auxiliary ones constructed in the induction of P1.

PATH P1. There are edge-disjoint minimum paths from each $s_{i}$ to a node in $\overline{S_{0} S_{r-1}} \cup$ $\overline{S_{0} S_{-(r-1)}}$. A distinct $s_{i}$ connects to a distinct node in $\overline{S_{0} S_{r-1}} \cup \overline{S_{0} S_{-(r-1)}}$.

PATH P2. There are edge-disjoint minimum paths from each $t_{i}$ to a node in $\overline{T_{r-1} T_{-(r-1)}}$. A distinct $t_{i}$ connects to a distinct node in $\overline{T_{r-1} T_{-(r-1)}}$.

PATH P3. There are edge-disjoint minimum paths from each of the nodes in $\overline{S_{0} S_{r-1}} \cup$ 
$\overline{S_{0} S_{-(r-1)}}$ assigned in Path P1 to the corresponding node in $\overline{T_{r-1} T_{-(r-1)}}$ assigned in Path P2.

We describe the construction of the three parts in the following.

- The construction of Path P1.

We prove the following argument using induction on $k, k \leq r-1$ : There are edge-disjoint minimum paths from each of the nodes in $\bigcup_{j=1}^{k} s_{j} \cup \overline{S_{k} S_{-k}}$ to a node in $\overline{S_{0} S_{k}} \cup \overline{S_{0} S_{-k}}$. It is easy to check that the argument holds when $k=1$, since $s_{1}$ is on $S_{0}$, and $S_{1}$ and $S_{-1}$ are already on $\overline{S_{0} S_{1}} \cup \overline{S_{0} S_{-1}}$. Assume that the argument holds when $k=i$. Since $s_{i+1}$ is on $\overline{S_{i} S_{-i}}$, by the induction hypothesis, there is a path from $s_{i+1}$ to a node on $\overline{S_{0} S_{i}} \cup \overline{S_{0} S_{-i}}$. The nodes $S_{i+1}$ and $S_{-(i+1)}$ are already on $\overline{S_{0} S_{i+1}} \cup \overline{S_{0} S_{-(i+1)}}$. The paths constructed in the induction hypothesis for the nodes on $\overline{S_{i}\left(s_{i+1}+[[1,-1]]\right)}$ can be extended horizontally to the nodes on $\overline{\left(S_{i+1}+[[-1,1]]\right)\left(s_{i+1}+[[0,-1]]\right)}$. Similarly, the paths constructed in the induction hypothesis for the nodes on $\overline{S_{-i}\left(s_{i+1}+[[-1,1]]\right)}$ can be extended vertically to the nodes on $\overline{\left(S_{-(i+1)}+[[1,-1]]\right)\left(s_{i+1}+[[-1,0]]\right)}$. Thus, the argument holds when $k=i+1$. This completes the induction for the argument. Since $s_{r}$ is on $\overline{S_{r-1} S_{-(r-1)}}$, the construction of Path P1 is completed.

- The construction of Path P2.

We use induction on $r$. If $r=1$, it is clear that $t_{1}$ is on $\overline{T_{0} T_{0}}$. Assume that Path $\mathrm{P} 2$ holds when $r=i$. The node $t_{i+1}$ is already on the line $\overline{T_{i} T_{-i}}$. The paths for the nodes on $\overline{T_{i-1}\left(t_{i+1}+[[1,0]]\right)}$ constructed in the induction hypothesis can be extended vertically to the nodes on $\overline{T_{i}\left(t_{i+1}+[[1,-1]]\right)}$. Similarly, the paths for the nodes on $\overline{T_{-(i-1)}\left(t_{i+1}+[[0,1]]\right)}$ constructed in the induction hypothesis can be extended horizontally to the nodes on $\overline{T_{-i}\left(t_{i+1}+[[-1,1]]\right)}$. Thus, the induction step is completed, and Path P2 follows.

- The construction of Path P3.

The nodes on $\overline{S_{0} S_{r-1}}$ that are assigned to $s_{i} \mathrm{~s}$ in Path $\mathrm{P} 1$ can be routed right then down to the corresponding nodes on $\overline{T_{r-1} T_{-(r-1)}}$ that are assigned to the $t_{i}$ 's in Path P2. Similarly, the nodes on $\overline{S_{-1} S_{-(r-1)}}$ that are assigned to the $s_{i}$ 's in Path P1 can be routed down then right to the corresponding nodes on $\overline{T_{r-1} T_{-(r-1)}}$ that are assigned to the $t_{i}$ 's in Path P2. It is simple to see that the paths are edge-disjoint.

Theorem 1 follows immediately by Lemmas 1 and 2 .

Now consider the the path coloring problem [7] in an on-line and distributed setting. In this problem we must color the paths assigned in the on-line source routing problem studied in our paper such that any two packets that share an edge have different colors. Note that the congestion is a lower bound for the number of colors used in the path coloring problem. By Lemma 1, the number of colors used in path coloring algorithms must be $\Omega(m \log N)$. Also note that the paths assigned in Lemma 2 for the adversary can be colored with $m$ colors. As a result, we have the following corollary.

COROLLARY 1. The competitive ratio of any on-line source routing and coloring algorithms on $N \times N$ tori is $\Omega(\log N)$. 
4. A Deterministic Algorithm. In this section we give a deterministic on-line source routing algorithm named COUNTER on $N \times N$ tori and show that its competitive ratio is $O(\log N)$. Consider an input $I=\delta_{1} \delta_{2} \cdots \delta_{m}$ of length $m$ and a packet $\delta_{k}$ for some $k$. The path assigned to $\delta_{k}$ depends only on the packets in $\delta_{1} \delta_{2} \cdots \delta_{k-1}$ that are equivalent to $\delta_{k}$. That is, two nonequivalent packets are processed by COUNTER independently. (Note that in this way the path for a packet $\delta=\langle\langle s, t\rangle\rangle$ is independent of packets whose source nodes are not $s$. So COUNTER is a distributed source routing algorithm.) As a result, in the following we focus on packets from node $s$ to node $t$, for some nodes $s$ and $t$.

Given an input of many equivalent packets from $s$ to $t$, COUNTER employs the locally optimal heuristic that distributes the loads evenly among the intermediate edges and nodes from $s$ to $t$. Furthermore, COUNTER maintains even loads for each incoming packet in an on-line manner with the following strategy.

Let $d$ be the distance from $s$ to $t$ and let $\delta$ be a packet from $s$ to $t$. We label the nodes in $S V_{\delta}(i), 1 \leq i \leq d+1$, as $\langle i, 1\rangle,\langle i, 2\rangle, \ldots,\left\langle i,\left|S V_{\delta}(i)\right|\right\rangle$ in the way that the $x$-coordinate of the distance vector from $s$ to $\langle i, j\rangle$ is larger than that from $s$ to $\langle i, j+1\rangle$, $1 \leq j<\left|S V_{\delta}(i)\right|$. COUNTER keeps a counter for each node $\langle i, j\rangle$. We use $C_{k}(i, j)$ to denote, after $k$ packets from $s$ to $t$ are processed, the number of packets that cross $\langle i, j\rangle$. These counters are stored on node $s$. Let $\delta$ be the packet we currently consider and assume that $\delta$ is the $(k+1)$ th packet. COUNTER reserves a path for $\delta$ hop by hop in the following way: Assume that the path has been constructed to a node $u \in S V_{\delta}(i)$. In general, $u$ connects to two nodes in the next slice, say, $\langle i+1, j\rangle$ and $\langle i+1, j+1\rangle$. If $C_{k}(i+1, j) \leq C_{k}(i+1, j+1)$, then the path is extended to $\langle i+1, j\rangle$. Otherwise, the path is extended to $\langle i+1, j+1\rangle$. In the boundaries, $u$ connects to only one node in the next slice. In this case the path is extended to that node. This path reservation process is carried out in the source node $s$, based on the counters saved on $s$.

As an example, Figure 4 shows the counter values after nine packets are processed. The nodes on a dashed line are in the same slice. We will show in Lemmas 3 and 4 that COUNTER has the property that it distributes the packets evenly among the nodes in a

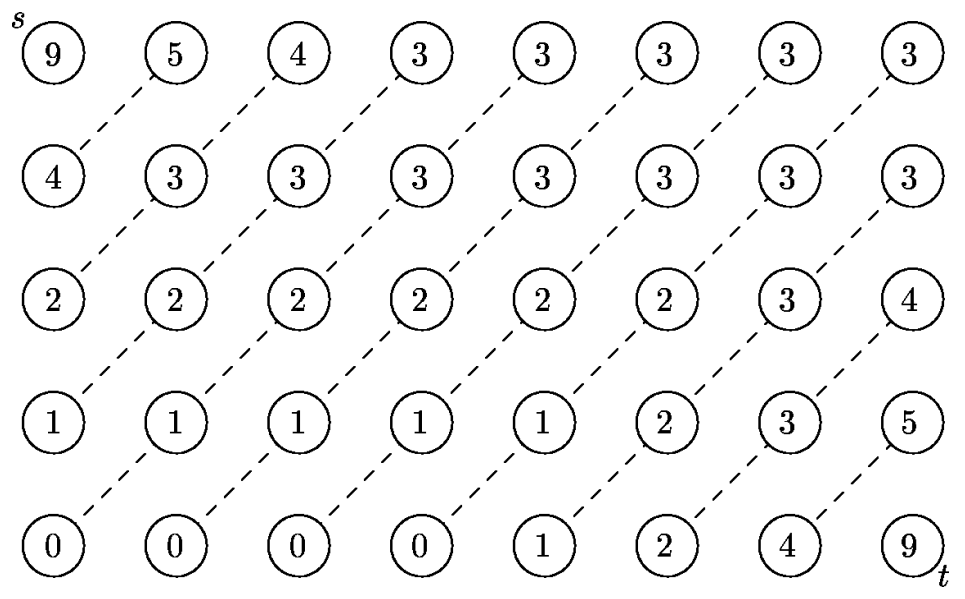

Fig. 4. The counter values after nine packets are processed. 
slice, and COUNTER maintains this property in an on-line manner in that the property is true from packet to packet.

LEMMA 3. $C_{k}(i, j)-C_{k}(i, j+1)=0$ or 1 , for any $k, i$, and $j$, where $1 \leq i \leq d+1$ and $1 \leq j<\left|S V_{\delta}(i)\right|$.

ProOF. The slices from $s$ to $t$ form a rectangle as in Figure 4. For slices that are close to $s,\left|S V_{\delta}(i+1)\right|=\left|S V_{\delta}(i)\right|+1$. For the middle slices, $\left|S V_{\delta}(i+1)\right|=\left|S V_{\delta}(i)\right|$. For slices that are close to $t,\left|S V_{\delta}(i+1)\right|=\left|S V_{\delta}(i)\right|-1$. We refer to these three regions as the first, the second, and the third regions, respectively. Formally, let $q$ be the largest integer such that $\left|S V_{\delta}(q)\right|=q$. We say that slice $i$ is in the first region if $1 \leq i \leq q$. Slice $i$ is in the second region if $\left|S V_{\delta}(i)\right|=\left|S V_{\delta}(q)\right|$. Slice $i$ is in the third region if $d+2-q \leq i \leq d+1$. Note that by this definition some slices may belong to multiple regions. For example, slice $q=5$ in Figure 4 is the last slice in the first region and the first slice in the second region.

First we show that the lemma is true for slices in the first region. We will show that the lemma holds for slices in the second and third regions at the end of the proof.

We use induction on $k$ on the following three hypotheses:

H1. $C_{k}(i, j)-C_{k}(i, j+1)=0$ or 1 .

H2. If $C_{k}(i, j)-C_{k}(i, j+l)=0$ for some $l \geq 0$, then $C_{k}(i+1, j)-C_{k}(i+1, j+l+1)=$ 0 or 1 .

H3. If $C_{k}(i, j)-C_{k}(i, j+l)=l$ for some $l \geq 0$, then $C_{k}(i+1, j)-C_{k}(i+1, j+l+1)=l$ or $l+1$.

It is trivial to see that the hypotheses hold for all $i$ and $j$ initially, because all counters are 0 . Assume that the hypotheses hold after $k_{0}$ packets are processed. In the following we show that after a new packet (the $\left(k_{0}+1\right)$ th packet) is processed the hypotheses still hold. For convenience, let $C(i, j)=C_{k_{0}}(i, j)$ and $C^{\prime}(i, j)=C_{k_{0}+1}(i, j)$.

First we show by contradiction that $\mathrm{H} 1$ is true after the new packet is processed. Assume that $\langle i, j\rangle$ is the node with the smallest slice number $i$ whose counter value changes (i.e., the new packet goes through it) and that the change violates H1 (between $\langle i, j\rangle$ and $\langle i, j-1\rangle$ or between $\langle i, j\rangle$ and $\langle i, j+1\rangle)$. If $\langle i, j\rangle$ is the first node in the slice, i.e., $j=1$, then $C(i, 1)-C(i, 2)$ must be 0 , or COUNTER will choose $\langle i, 2\rangle$ to route the new packet. In this case, $C^{\prime}(i, 1)-C^{\prime}(i, 2)=1, \mathrm{H} 1$ is not violated. Similarly, if $\langle i, j\rangle$ is the last node in the slice, i.e., $j=i$, then $C(i, i-1)-C(i, i)$ must be 1 . In this case, $C^{\prime}(i, i-1)-C^{\prime}(i, i)=0, \mathrm{H} 1$ is not violated, either. Suppose $1<j<i$. We consider the following two cases, H1-1 and H1-2. In the first case we consider the situation that the new packet visits $\langle i-1, j-1\rangle$ before it visits $\langle i, j\rangle$. In the other case we consider the situation that the new packet visits $\langle i-1, j\rangle$ before it visits $\langle i, j\rangle$. These two cases are all that we need to verify hypothesis $\mathrm{H} 1$.

- Case H1-1. The new packet comes from $\langle i-1, j-1\rangle$. See Figure 5(a). In the figure the shaded nodes are where the new packet goes through. The dashed curves and the associated numbers indicate the counter differences and their changes. For example, $1 \rightarrow 0$ between $\langle i, j-1\rangle$ and $\langle i, j\rangle$ indicates that $C(i, j-1)-C(i, j)=1$ and $C^{\prime}(i, j-1)-C^{\prime}(i, j)=0$. The nodes on a dashed line are in the same slice. In this 


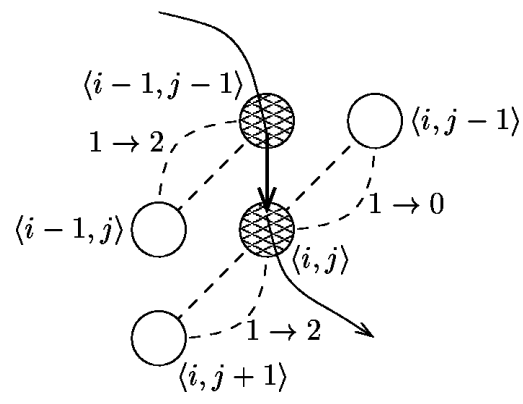

(a)

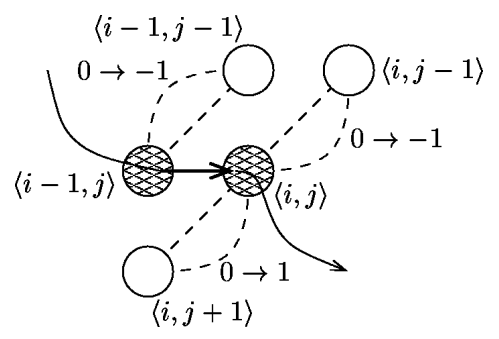

(b)

Fig. 5. Cases considered in the induction step of H1. (a) Case H1-1, (b) Case H1-2.

case, $C(i, j-1)-C(i, j)$ must be 1 , or COUNTER will choose $\langle i, j-1\rangle$ to route the new packet. Because we want $\mathrm{H} 1$ be violated at $\langle i, j\rangle$ after the processing of the new packet, $C(i, j)-C(i, j+1)$ must be 1 so that $C^{\prime}(i, j)-C^{\prime}(i, j+1)=2$. If $C(i-1, j-$ 1) $-C(i-1, j)=0$, then by the induction hypothesis $\mathrm{H} 2, C(i, j-1)-C(i, j+1)$ must be 0 or 1 . This is not what we have here. So $C(i-1, j-1)-C(i-1, j)=1$. Because the new packet is routed through $\langle i-1, j-1\rangle, C^{\prime}(i-1, j-1)-C^{\prime}(i-1, j)=2$. Here $\langle i-1, j-1\rangle$ is a node with smaller slice number $i-1$ that violates $\mathrm{H} 1$, a contradiction.

- Case H1-2. The new packet comes from $\langle i-1, j\rangle$. See Figure 5(b). In this case, $C(i, j)-C(i, j+1)=0$. Because we want $\mathrm{H} 1$ to be violated at $\langle i, j\rangle$ after the processing of the new packet, $C(i, j-1)-C(i, j)$ must be 0 . If $C(i-1, j-1)-$ $C(i-1, j)=1$, then by the induction hypothesis H3, $C(i, j-1)-C(i, j+1)$ must be 1 or 2 . This is not what we have here. So $C(i-1, j-1)-C(i-1, j)=0$. Because the new packet is routed through $\langle i-1, j\rangle, C^{\prime}(i-1, j-1)-C^{\prime}(i-1, j)=-1$. Here $\langle i-1, j\rangle$ is a node with the smaller slice number $i-1$ that violates $\mathrm{H} 1$, a contradiction.

In the two cases we proved that there exists a node with slice number $i-1$ that $\mathrm{H} 1$ is violated around it. However, it is easy to check that $\mathrm{H} 1$ is true at nodes in slices 2 and 3 after any number of packets are processed. (The reader can try as few as six packets and find this.) As a result, the contradiction is established and $\mathrm{H} 1$ holds after the new packet is processed and we have the following observation.

OBSERVATION 1. Assume that the new packet goes through $\langle i, j\rangle$. Then the difference of the counter values of $\langle i, j-1\rangle$ and $\langle i, j\rangle$ (if $\langle i, j-1\rangle$ exists) changes from 1 to 0 . Similarly, the difference of the counter values of $\langle i, j\rangle$ and $\langle i, j+1\rangle($ if $\langle i, j+1\rangle$ exists) changes from 0 to 1 . Formally,

$$
\begin{aligned}
C(i, j-1)-C(i, j) & =1, & & \text { if } \quad j>1, \\
C^{\prime}(i, j-1)-C^{\prime}(i, j) & =0, & & \text { if } \quad j>1, \\
C(i, j)-C(i, j+1) & =0, & & \text { if } j<i, \\
C^{\prime}(i, j)-C^{\prime}(i, j+1) & =1, & & \text { if } \quad j<i .
\end{aligned}
$$


In the following we show that $\mathrm{H} 2$ is true after the new packet is processed. Fix some $i, j$, and $l$ such that

$$
C^{\prime}(i, j)-C^{\prime}(i, j+l)=0,
$$

we show in the following that $C^{\prime}(i+1, j)-C^{\prime}(i+1, j+l+1)=0$ or 1 . We consider all the cases where the new packet visits a node in the node set $\overline{\langle i+1, j\rangle\langle i+1, j+l+1\rangle}$. (Recall that $\overline{A B}$ is the set of nodes on the imaginary line from node $A$ to node $B$, inclusive.) For the other cases where the nodes in $\overline{\langle i+1, j\rangle\langle i+1, j+l+1\rangle}$ are not visited, it is easy to see that the nodes in $\overline{\langle i, j\rangle\langle i, j+l\rangle}$ are not visited, either. Therefore the counter values of the nodes in $\overline{\langle i, j\rangle\langle i, j+l\rangle} \cup \overline{\langle i+1, j\rangle\langle i+1, j+l+1\rangle}$ are not changed, which implies that $C(i, j)-C(i, j+l)=C^{\prime}(i, j)-C^{\prime}(i, j+l)$ and $C(i+$ $1, j)-C(i+1, j+l+1)=C^{\prime}(i+1, j)-C^{\prime}(i+1, j+l+1)$. By our assumption in (2), $C(i, j)-C(i, j+l)=C^{\prime}(i, j)-C^{\prime}(i, j+l)=0$. By the induction hypothesis, $C(i+1, j)-C(i+1, j+l+1)=0$ or 1 . Therefore $C^{\prime}(i+1, j)-C^{\prime}(i+1, j+l+1)=0$ or 1 .

- Case H2-1. The new packet visits node $\langle i, j-1\rangle$ and node $\langle i+1, j\rangle$. See Figure 6 . The nodes in the shaded area, that is, nodes in $\overline{\langle i, j\rangle\langle i, j+l\rangle} \cup \overline{\langle i+1, j\rangle\langle i+1, j+l+1\rangle}$, are those that we are concerned with in the induction. By (1c) on nodes $\langle i, j-1\rangle$ and $\langle i, j\rangle$,

$$
C(i, j-1)-C(i, j)=0 .
$$

By (1a) on nodes $\langle i+1, j-1\rangle$ and $\langle i+1, j\rangle$,

$$
C(i+1, j-1)-C(i+1, j)=1 .
$$

By (1c) on nodes $\langle i+1, j\rangle$ and $\langle i+1, j+1\rangle$,

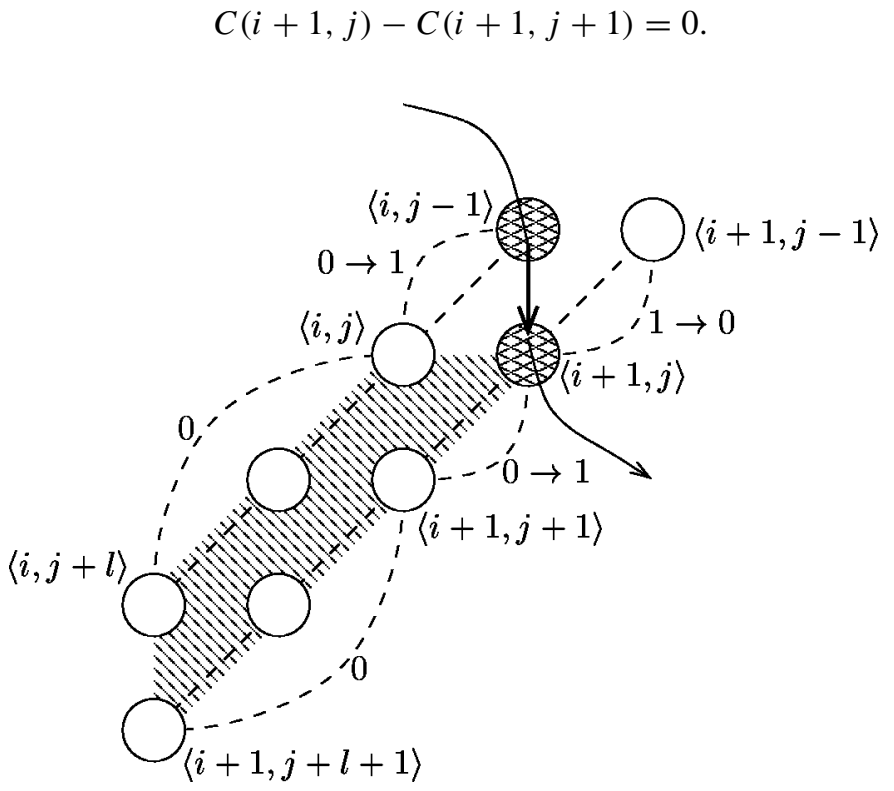

Fig. 6. Case H2-1 in the induction step of $\mathrm{H} 2$. 
By (1d) on nodes $\langle i+1, j\rangle$ and $\langle i+1, j+1\rangle$,

$$
C^{\prime}(i+1, j)-C^{\prime}(i+1, j+1)=1 .
$$

Because the new packet does not visit nodes in $\overline{\langle i, j\rangle\langle i, j+l\rangle}$,

$$
C(i, j)-C(i, j+l)=C^{\prime}(i, j)-C^{\prime}(i, j+l) .
$$

Also the new packet does not visit nodes in $\overline{\langle i+1, j+1\rangle\langle i+1, j+l+1\rangle}$, so

$$
\begin{aligned}
& C(i+1, j+1)-C(i+1, j+l+1) \\
& =C^{\prime}(i+1, j+1)-C^{\prime}(i+1, j+l+1) .
\end{aligned}
$$

By (2), (3), and (7),

$$
C(i, j-1)-C(i, j+l)=0 .
$$

By the induction hypothesis $\mathrm{H} 2$,

$$
C(i+1, j-1)-C(i+1, j+l+1)=0 \text { or } 1 .
$$

By (4) and (5),

$$
C(i+1, j+1)-C(i+1, j+l+1)=0 .
$$

By (8),

$$
C^{\prime}(i+1, j+1)-C^{\prime}(i+1, j+l+1)=0 .
$$

By (6),

$$
C^{\prime}(i+1, j)-C^{\prime}(i+1, j+l+1)=1 .
$$

- Case H2-2. The new packet visits node $\langle i, j+h\rangle, 0 \leq h<l$, then node $\langle i+1, j+h\rangle$ or node $\langle i+1, j+h+1\rangle$ in slice $i+1$. By (1d), $C^{\prime}(i, j+h)-C^{\prime}(i, j+h+1)=1$. Therefore $C^{\prime}(i, j)-C^{\prime}(i, j+l) \geq 1$, which violates our assumption in (2). We ignore this case.

- Case H2-3. The new packet visits $\langle i, j+l\rangle$ and $\langle i+1, j+l\rangle$. See Figure 7. By Observation 1,

$$
\begin{aligned}
C^{\prime}(i, j+l-1)-C^{\prime}(i, j+l) & =0, \\
C(i+1, j+l-1)-C(i+1, j+l) & =1, \\
C^{\prime}(i+1, j+l-1)-C^{\prime}(i+1, j+l) & =0, \\
C^{\prime}(i+1, j+l)-C^{\prime}(i+1, j+l+1) & =1 .
\end{aligned}
$$

Because the new packet does not visit nodes in $\overline{\langle i, j\rangle\langle i, j+l-1\rangle} \cup$ $\overline{\langle i+1, j\rangle\langle i+1, j+l-1\rangle}$,

(14) $C(i+1, j)-C(i+1, j+l-1)=C^{\prime}(i+1, j)-C^{\prime}(i+1, j+l-1)$. 


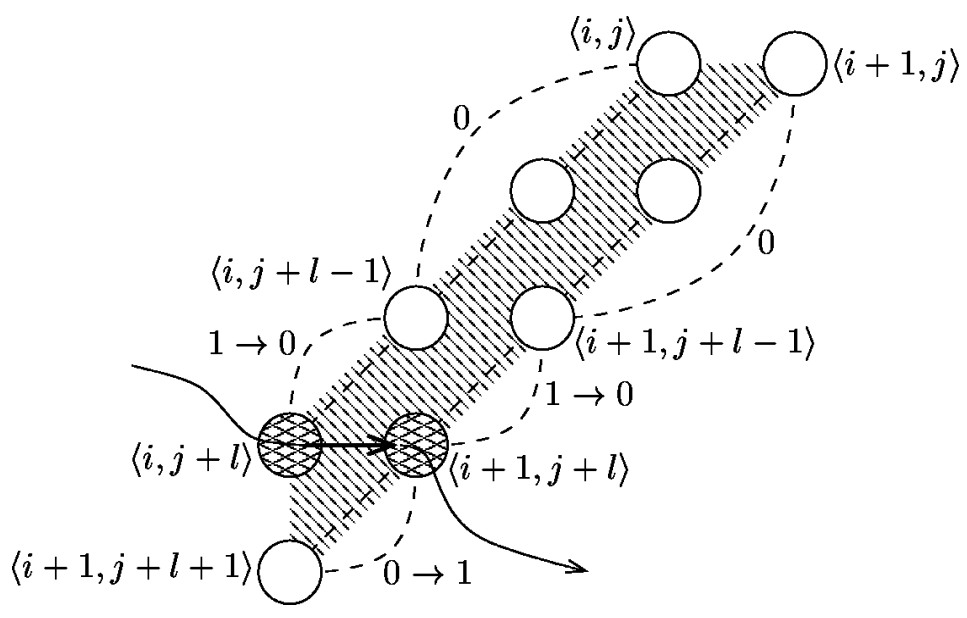

Fig. 7. Case H2-3 in the induction step of $\mathrm{H} 2$.

By (2) and (9),

$$
C^{\prime}(i, j)-C^{\prime}(i, j+l-1)=0 .
$$

By (13),

$$
C(i, j)-C(i, j+l-1)=0 .
$$

By the induction hypothesis $\mathrm{H} 2$,

$$
C(i+1, j)-C(i+1, j+l)=0 \text { or } 1 .
$$

By (10),

$$
C(i+1, j)-C(i+1, j+l-1)=0 .
$$

By (14),

$$
C^{\prime}(i+1, j)-C^{\prime}(i+1, j+l-1)=0 .
$$

By (11) and (12),

$$
C^{\prime}(i+1, j)-C^{\prime}(i+1, j+l+1)=1 .
$$

- Case H2-4. The new packet visits $\langle i, j+l\rangle$ and $\langle i+1, j+l+1\rangle$. See Figure 8. By Observation 1,

$$
\begin{aligned}
C^{\prime}(i, j+l-1)-C^{\prime}(i, j+l) & =0, \\
C^{\prime}(i+1, j+l)-C^{\prime}(i+1, j+l+1) & =0 .
\end{aligned}
$$

By (2) and (15),

$$
C^{\prime}(i, j)-C^{\prime}(i, j+l-1)=0
$$




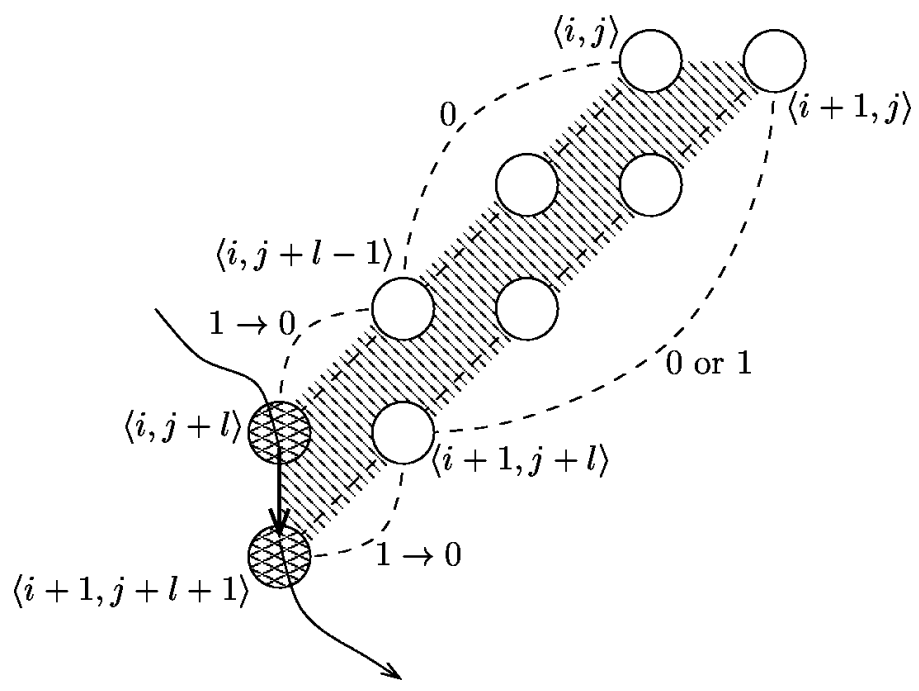

Fig. 8. Case $\mathrm{H} 2-4$ in the induction step of $\mathrm{H} 2$.

Because the new packet does not visit nodes in $\overline{\langle i, j\rangle\langle i, j+l-1\rangle}$,

$$
C(i, j)-C(i, j+l-1)=C^{\prime}(i, j)-C^{\prime}(i, j+l-1)=0 .
$$

By the induction hypothesis $\mathrm{H} 2$,

$$
C(i+1, j)-C(i+1, j+l)=0 \text { or } 1 .
$$

Because the new packet does not visit nodes in $\overline{\langle i+1, j\rangle\langle i+1, j+l\rangle}$,

$$
C^{\prime}(i+1, j)-C^{\prime}(i+1, j+l)=C(i+1, j)-C(i+1, j+l)=0 \text { or } 1 .
$$

By (16),

$$
C^{\prime}(i+1, j)-C^{\prime}(i+1, j+l+1)=0 \text { or } 1 .
$$

- Case H2-5. The new packet visits $\langle i, j+l+1\rangle$ and $\langle i+1, j+l+1\rangle$. See Figure 9 . By Observation 1,

$$
\begin{aligned}
C(i+1, j+l)-C(i+1, j+l+1) & =1, \\
C^{\prime}(i+1, j+l)-C^{\prime}(i+1, j+l+1) & =0 .
\end{aligned}
$$

Because the new packet does not visit nodes in $\overline{\langle i+1, j\rangle\langle i+1, j+l\rangle}$,

$$
C(i+1, j)-C(i+1, j+l)=C^{\prime}(i+1, j)-C^{\prime}(i+1, j+l) .
$$

By the induction hypothesis $\mathrm{H} 2$,

$$
C(i+1, j)-C(i+1, j+l+1)=0 \text { or } 1 .
$$




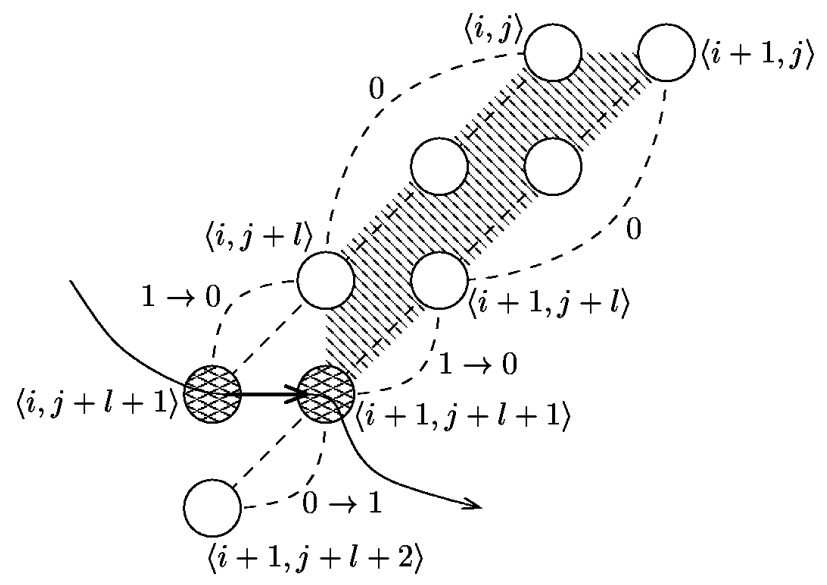

Fig. 9. Case H2-5 in the induction step of $\mathrm{H} 2$.

By (17),

$$
C(i+1, j)-C(i+1, j+l)=0 \text { or }-1 .
$$

By the hypothesis $\mathrm{H} 1, C(i+1, j) \geq C(i+1, j+1) \geq C(i+1, j+2) \geq \cdots$. Therefore,

$$
C(i+1, j)-C(i+1, j+l)=0
$$

By (19),

$$
C^{\prime}(i+1, j)-C^{\prime}(i+1, j+l)=0
$$

By (18),

$$
C^{\prime}(i+1, j)-C^{\prime}(i+1, j+l+1)=0 .
$$

Thus far we have considered all cases where the new packet visits a node in the node set $\overline{\langle i+1, j\rangle\langle i+1, j+l+1\rangle}$ and we proved that

$$
C^{\prime}(i+1, j)-C^{\prime}(i+1, j+l+1)=0 \text { or } 1
$$

in all the cases except in case H2-2 where our assumption in (2) is violated in the first place. Therefore the induction step for hypothesis $\mathrm{H} 2$ is complete.

In the following we show that $\mathrm{H} 3$ is true after the new packet is processed. Fix some $i, j$, and $l$ such that

$$
C^{\prime}(i, j)-C^{\prime}(i, j+l)=l,
$$

we show that $C^{\prime}(i+1, j)-C^{\prime}(i+1, j+l+1)=l$ or $l+1$ by considering all the cases where the new packet visits a node in the node set $\overline{\langle i+1, j\rangle\langle i+1, j+l+1\rangle}$. For the other cases where the nodes in $\overline{\langle i+1, j\rangle\langle i+1, j+l+1\rangle}$ are not visited, it is easy to see that the nodes in $\overline{\langle i, j\rangle\langle i, j+l\rangle}$ are not visited either. Therefore the counter values of the nodes in $\overline{\langle i, j\rangle\langle i, j+l\rangle} \cup \overline{\langle i+1, j\rangle\langle i+1, j+l+1\rangle}$ are not changed, which implies that $C(i, j)-C(i, j+l)=C^{\prime}(i, j)-C^{\prime}(i, j+l)$ and $C(i+1, j)-$ 


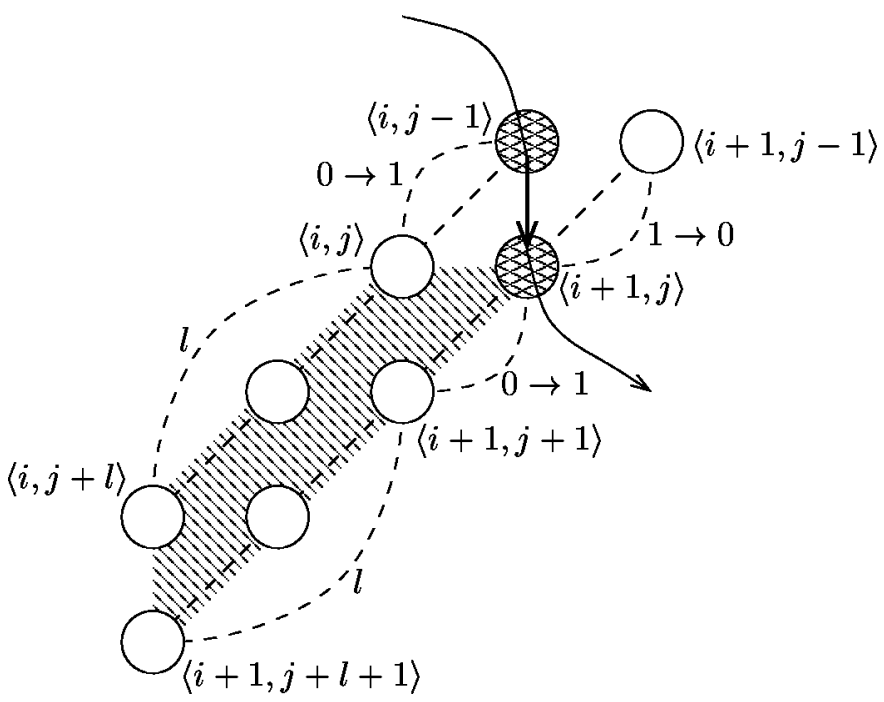

Fig. 10. Case H3-1 in the induction step of H3.

$C(i+1, j+l+1)=C^{\prime}(i+1, j)-C^{\prime}(i+1, j+l+1)$. By our assumption in (20), $C(i, j)-C(i, j+l)=C^{\prime}(i, j)-C^{\prime}(i, j+l)=l$. By the induction hypothesis, $C(i+1, j)-C(i+1, j+l+1)=l$ or $l+1$. Therefore $C^{\prime}(i+1, j)-C^{\prime}(i+1, j+l+1)=l$ or $l+1$.

- Case H3-1. The new packet visits node $\langle i, j-1\rangle$ and then node $\langle i+1, j\rangle$. See Figure 10. By Observation 1,

$$
\begin{aligned}
& C(i+1, j)-C(i+1, j+1)=0, \\
& C(i+1, j)-C(i+1, j+1)=1 .
\end{aligned}
$$

Because the new packet does not visit nodes in $\overline{\langle i+1, j+1\rangle\langle i+1, j+l+1\rangle}$,

$$
\begin{aligned}
C(i+1, j+1)-C(i+1, j+l+1) \\
\quad=C^{\prime}(i+1, j+1)-C^{\prime}(i+1, j+l+1) .
\end{aligned}
$$

By the induction hypothesis $\mathrm{H} 3$,

$$
C(i+1, j)-C(i+1, j+l+1)=l \text { or } l+1 .
$$

By (21),

$$
C(i+1, j+1)-C(i+1, j+l+1)=l \text { or } l+1 .
$$

By the hypothesis H1, $C(i+1, j+1)-C(i+1, j+l+1)=\sum_{j^{\prime}=j+1}^{j+l}\left(C\left(i+1, j^{\prime}\right)-\right.$ $\left.C\left(i+1, j^{\prime}+1\right)\right) \leq \sum_{j^{\prime}=j+1}^{j+l}(1)=l$, therefore,

$$
C(i+1, j+1)-C(i+1, j+l+1)=l .
$$




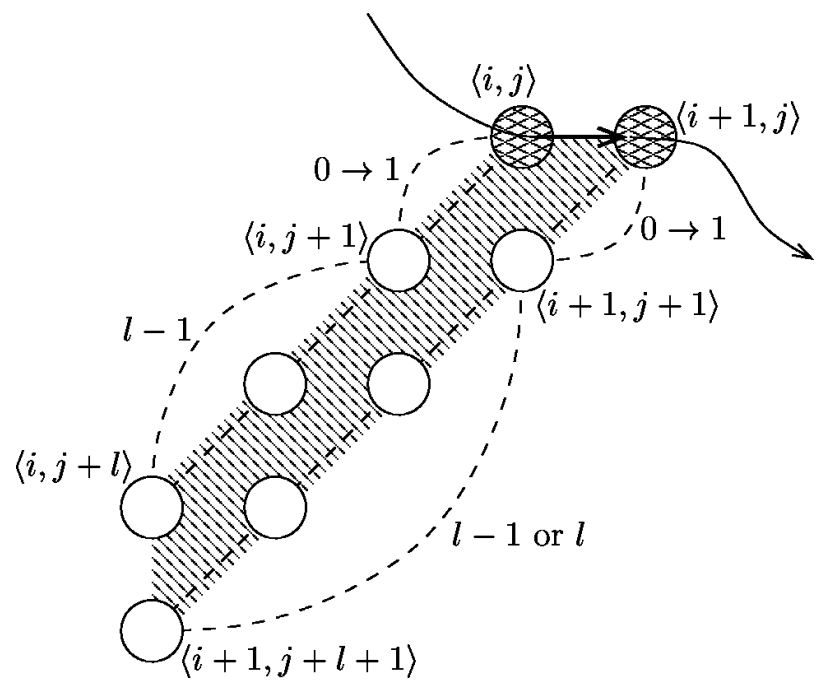

Fig. 11. Case H3-2 in the induction step of H3.

By (23),

$$
C^{\prime}(i+1, j+1)-C^{\prime}(i+1, j+l+1)=l .
$$

By (22),

$$
C^{\prime}(i+1, j)-C^{\prime}(i+1, j+l+1)=l+1 .
$$

- Case H3-2. The new packet visits $\langle i, j\rangle$ and $\langle i+1, j\rangle$. See Figure 11. By Observation 1 ,

$$
\begin{aligned}
C^{\prime}(i, j)-C^{\prime}(i, j+1) & =1, \\
C^{\prime}(i+1, j)-C^{\prime}(i+1, j+1) & =1 .
\end{aligned}
$$

Because the new packet does not visit nodes in $\overline{\langle i, j+1\rangle\langle i, j+l\rangle} \cup \overline{\langle i+1, j+1\rangle}$ $\overline{\langle i+1, j+l+1\rangle}$,

$$
\begin{aligned}
& C(i, j+1)-C(i, j+l)=C^{\prime}(i, j+1)-C^{\prime}(i, j+l) \\
& C(i+1, j+1)-C(i+1, j+l+1) \\
& =C^{\prime}(i+1, j+1)-C^{\prime}(i+1, j+l+1) .
\end{aligned}
$$

By (20) and (24),

$$
C^{\prime}(i, j+1)-C^{\prime}(i, j+l)=l-1 \text {. }
$$

By (26),

$$
C(i, j+1)-C(i, j+l)=l-1 .
$$

By the induction hypothesis $\mathrm{H} 3$,

$$
C(i+1, j+1)-C(i+1, j+l+1)=l-1 \text { or } l .
$$




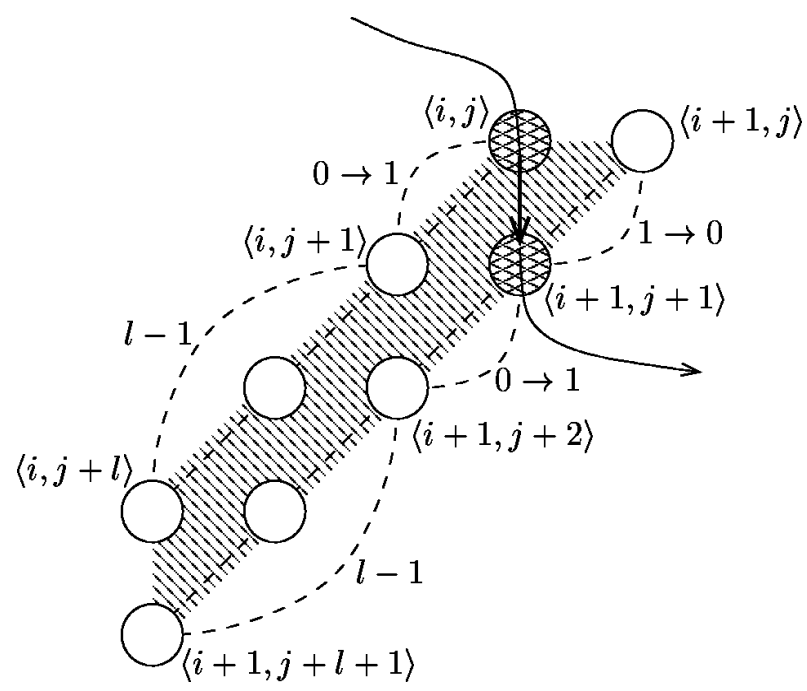

Fig. 12. Case H3-3 in the induction step of H3.

By (27),

$$
C^{\prime}(i+1, j+1)-C^{\prime}(i+1, j+l+1)=l-1 \text { or } l .
$$

By (25),

$$
C^{\prime}(i+1, j)-C^{\prime}(i+1, j+l+1)=l \text { or } l+1 .
$$

- Case H3-3. The new packet visits $\langle i, j\rangle$ and $\langle i+1, j+1\rangle$. See Figure 12. By Observation 1 ,

$$
\begin{aligned}
C^{\prime}(i, j)-C^{\prime}(i, j+1) & =1, \\
C^{\prime}(i+1, j)-C^{\prime}(i+1, j+1) & =0, \\
C(i+1, j+1)-C(i+1, j+2) & =0, \\
C^{\prime}(i+1, j+1)-C^{\prime}(i+1, j+2) & =1 .
\end{aligned}
$$

Because the new packet does not visit nodes in $\overline{\langle i, j+1\rangle\langle i, j+l\rangle} \cup \overline{\langle i+1, j+2\rangle}$ $\overline{\langle i+1, j+l+1\rangle}$,

$$
\begin{aligned}
& C(i, j+1)-C(i, j+l)=C^{\prime}(i, j+1)-C^{\prime}(i, j+l), \\
& \begin{array}{r}
C(i+1, j+2)-C(i+1, j+l+1) \\
=C^{\prime}(i+1, j+2)-C^{\prime}(i+1, j+l+1) .
\end{array}
\end{aligned}
$$

By (20) and (28),

$$
C^{\prime}(i, j+1)-C^{\prime}(i, j+l)=l-1 .
$$


By (32),

$$
C(i, j+1)-C(i, j+l)=l-1 .
$$

By the induction hypothesis $\mathrm{H} 3$,

$$
C(i+1, j+1)-C(i+1, j+l+1)=l-1 \text { or } l .
$$

By (30),

$$
C(i+1, j+2)-C(i+1, j+l+1)=l-1 .
$$

By (33),

$$
C^{\prime}(i+1, j+2)-C^{\prime}(i+1, j+l+1)=l-1 .
$$

By (29) and (31),

$$
C^{\prime}(i+1, j)-C^{\prime}(i+1, j+l+1)=l .
$$

- Case H3-4. The new packet visits $\langle i, j+l-h\rangle, 0 \leq h<l$, then $\langle i+1, j+l-h\rangle$ or $\langle i+1, j+l-h+1\rangle$. By Observation $1, C^{\prime}(i, j+l-h-1)-C^{\prime}(i, j+l-h)=0$. Therefore $C^{\prime}(i, j)-C^{\prime}(i, j+l) \leq l-1$, which violates our assumption in (20). We ignore this case.

- Case H3-5. The new packet visits $\langle i, j+l+1\rangle$ and $\langle i+1, j+l+1\rangle$. See Figure 13. By Observation 1,

$$
\begin{aligned}
C(i, j+l)-C(i, j+l+1) & =1, \\
C(i+1, j+l)-C(i+1, j+l+1) & =1, \\
C^{\prime}(i+1, j+l)-C^{\prime}(i+1, j+l+1) & =0, \\
C(i+1, j+l+1)-C(i+1, j+l+2) & =0 .
\end{aligned}
$$

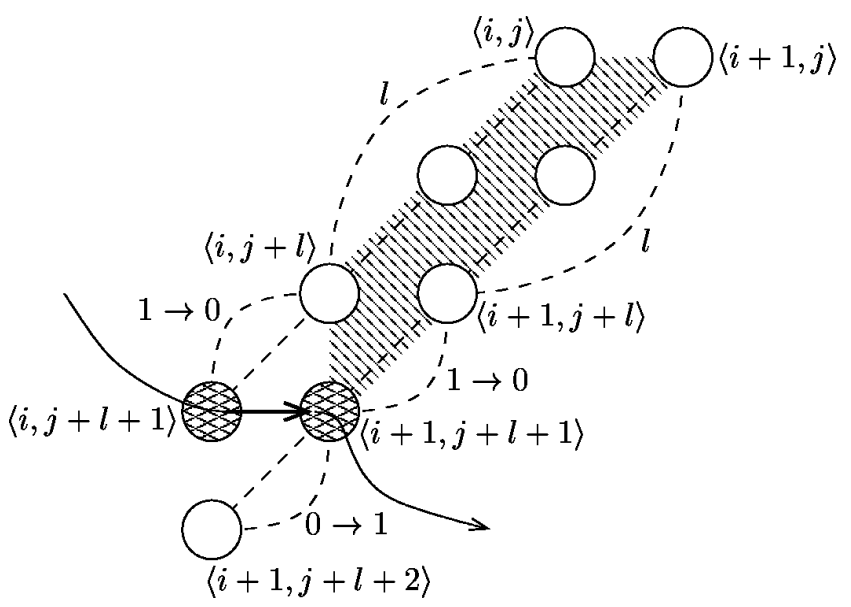

Fig. 13. Case H3-5 in the induction step of H3. 
Because the new packet does not visit nodes in $\overline{\langle i, j\rangle\langle i, j+l\rangle} \overline{\langle i+1, j\rangle\langle i+1, j+l\rangle}$,

$$
\begin{aligned}
C(i, j)-C(i, j+l) & =C^{\prime}(i, j)-C^{\prime}(i, j+l), \\
C(i+1, j)-C(i+1, j+l) & =C^{\prime}(i+1, j)-C^{\prime}(i+1, j+l) .
\end{aligned}
$$

By (20) and (38),

$$
C(i, j)-C(i, j+l)=l \text {. }
$$

By (34),

$$
C(i, j)-C(i, j+l+1)=l+1 .
$$

By the induction hypothesis $\mathrm{H} 3$,

$$
C(i+1, j)-C(i+1, j+l+2)=l+1 \text { or } l+2 .
$$

By (35) and (37),

$$
C(i+1, j)-C(i+1, j+l)=l .
$$

By (39),

$$
C^{\prime}(i+1, j)-C^{\prime}(i+1, j+l)=l \text {. }
$$

By (36),

$$
C^{\prime}(i+1, j)-C^{\prime}(i+1, j+l+1)=l .
$$

Thus far we have considered all cases where the new packet visits a node in the node set $\overline{\langle i+1, j\rangle\langle i+1, j+l+1\rangle}$ and we proved that

$$
C^{\prime}(i+1, j)-C^{\prime}(i+1, j+l+1)=l \text { or } l+1
$$

in all the cases except in case H3-4 where our assumption in (20) is violated in the first place. Therefore the induction step for hypothesis $\mathrm{H} 3$ is complete.

Now we have shown that the lemma is true for slices in the first region. In the following we will show that the lemma is true for slices in the second and third regions, too. First we give some intuition on what we will prove. Look at Figure 4, we claim:

ClaIM 1. Consider two nodes $\left\langle i_{1}, j\right\rangle$ and $\left\langle i_{2}, j\right\rangle$ in the second region. (Recall that for a slice $i$ in the second region, $\left|S V_{\delta}(i)\right|=\left|S V_{\delta}(q)\right|$, where slice $q$ is the last slice in the first region and also the first slice in the second region.) The counters of the two nodes are equal.

CLAIM 2. Consider a node $\langle i, j\rangle$ in the first region and its symmetric node $\langle d+2-i, j\rangle$ in the third region, the counters of the two nodes are equal.

It follows immediately that the lemma is true for nodes in the second and third regions. We prove in the following that the two claims are not coincidence and are true in general. 
First we prove Claim 1. We prove by induction on the number of packets processed so far on the argument: For any $j$, the counters of $\langle q, j\rangle$ and $\langle q+1, j\rangle$ are equal. (Assume that slice $q+1$ is in the second region, or there is nothing to prove.) Initially, the counters are 0 , so the argument is true. Assume that the argument is true after some number of packets are processed. Now consider a new packet and assume that it crosses $\langle q, j\rangle$, for some $j$. Slice $q$ is the last slice in the first region, so Observation 1 applies and

$$
\begin{array}{lll}
C(q, j-1)-C(q, j)=1, & \text { if } \quad\langle q, j-1\rangle \text { exists } \\
C(q, j)-C(q, j+1)=0, & \text { if } \quad\langle q, j+1\rangle \text { exists. }
\end{array}
$$

By the induction hypothesis,

$$
\begin{aligned}
C(q+1, j-1)-C(q+1, j)= & C(q, j-1)-C(q, j)=1, \\
& \text { if }\langle q+1, j-1\rangle \text { exists; } \\
C(q+1, j)-C(q+1, j+1)= & C(q, j)-C(q, j+1)=0, \\
& \text { if }\langle q+1, j+1\rangle \text { exists. }
\end{aligned}
$$

If $\langle q, j\rangle$ connects to $\langle q+1, j-1\rangle$ and $\langle q+1, j\rangle$ in the next slice, then by (40) COUNTER will choose $\langle q+1, j\rangle$ to route the new packet. If $\langle q, j\rangle$ connects to $\langle q+1, j\rangle$ and $\langle q+1, j+1\rangle$ in the next slice, then by (41) COUNTER will choose $\langle q+1, j\rangle$ to route the new packet. In both cases the new packet will be routed through $\langle q+1, j\rangle$. So the argument is true. A similar argument and proof can be constructed for slices $q+2, q+3$, $\ldots$ in the second region. So Claim 1 is true.

Next we prove Claim 2. We use induction on the number of packets processed so far to prove the following statement: For any $j$, the counters of $\langle q-1, j\rangle$ and $\langle d+2-(q-1), j\rangle$ are equal. (Slice $d+2-(q-1)$ is the second slice in the third region, and $\left|S V_{\delta}(d+2-(q-1))\right|=q-1$.) The statement is true initially, because all counters are 0 . Assume that the statement is true after a number of packets are processed. Now consider a new packet and assume that it crosses $\left\langle q-1, j_{1}\right\rangle,\left\langle q, j_{2}\right\rangle$, for some $j_{1}$ and $j_{2}$. By the proof for the second region, the new packet must cross $\left\langle d+2-q, j_{2}\right\rangle$. By Observation 1 a round $\left\langle q-1, j_{1}\right\rangle$,

$$
\begin{array}{lll}
C\left(q-1, j_{1}-1\right)-C\left(q-1, j_{1}\right)=1, & \text { if } \quad\left\langle q-1, j_{1}-1\right\rangle \text { exists; } \\
C\left(q-1, j_{1}\right)-C\left(q-1, j_{1}+1\right)=0, & \text { if } \quad\left\langle q-1, j_{1}+1\right\rangle \text { exists. }
\end{array}
$$

By the induction hypothesis,

$$
\begin{gathered}
C\left(d+2-(q-1), j_{1}-1\right)-C\left(d+2-(q-1), j_{1}\right) \\
=C\left(q-1, j_{1}-1\right)-C\left(q-1, j_{1}\right)=1, \\
\quad \text { if }\left\langle d+2-(q-1), j_{1}-1\right\rangle \text { exists; } \\
C\left(d+2-(q-1), j_{1}\right)-C\left(d+2-(q-1), j_{1}+1\right) \\
=C\left(q-1, j_{1}\right)-C\left(q-1, j_{1}+1\right)=0, \\
\text { if }\left\langle d+2-(q-1), j_{1}+1\right\rangle \text { exists. }
\end{gathered}
$$


$\left\langle q-1, j_{1}\right\rangle$ connects to $\left\langle q, j_{1}\right\rangle$ and $\left\langle q, j_{1}+1\right\rangle$ in the next slice, so $j_{2}$ must be $j_{1}$ or $j_{1}+1$. $\left\langle d+2-q, j_{2}\right\rangle$ connects to $\left\langle d+2-(q-1), j_{2}-1\right\rangle$ (if it exists) and $\langle d+2-(q-1)$, $\left.j_{2}\right\rangle$ (if it exists) in the next slice. If $j_{2}=j_{1}$, that is, $\left\langle d+2-q, j_{2}\right\rangle=\left\langle d+2-q, j_{1}\right\rangle$ connects to $\left\langle d+2-(q-1), j_{1}-1\right\rangle$ and $\left\langle d+2-(q-1), j_{1}\right\rangle$ in the next slice, then by (42) COUNTER will choose $\left\langle d+2-(q-1), j_{1}\right\rangle$ to route the new packet. If $j_{2}=j_{1}+1$, that is, $\left\langle d+2-q, j_{2}\right\rangle=\left\langle d+2-q, j_{1}+1\right\rangle$ connects to $\left\langle d+2-(q-1), j_{1}\right\rangle$ and $\left\langle d+2-(q-1), j_{1}+1\right\rangle$, then by (43) COUNTER will choose $\left\langle d+2-(q-1), j_{1}\right\rangle$ to route the new packet. In both cases the new packet crosses $\left\langle d+2-(q-1), j_{1}\right\rangle$. So the statement is true. A similar statement and proof can be applied to slices $d+2-(q-2)$, $d+2-(q-3), \ldots, d+1$. Claim 2 follows.

The three hypotheses, $\mathrm{H} 1, \mathrm{H} 2$, and $\mathrm{H} 3$, are true for all the slices in the three regions. Lemma 3 follows.

LEMMA 4. Assume that $k$ packets from $s$ to $t$ have been processed by COUNTER. Consider a slice of nodes $S V_{\delta}(i)$ and a node $v$ in that slice. The number of packets that travel through $v$ is less than $k /\left|S V_{\delta}(i)\right|+N$.

ProOF. By Lemma 3, $C(i, 1)-C\left(i,\left|S V_{\delta}(i)\right|\right) \leq\left|S V_{\delta}(i)\right|$. Thus the number of packets that travel through $v$ is no more than $k /\left|S V_{\delta}(i)\right|+\left|S V_{\delta}(i)\right|$. Note that the number of nodes in a slice is bounded above by the side length of the torus. So $k /\left|S V_{\delta}(i)\right|+\left|S V_{\delta}(i)\right|<$ $k /\left|S V_{\delta}(i)\right|+N$.

THEOREM 2. The competitive ratio of COUNTER on $N \times N$ tori is $O(\log N)$. Specifically, for any input I,

$$
\operatorname{cost}_{\text {COUNTER }}(I) \leq O(\log N) \times \operatorname{cost}_{\mathrm{ADV}}(I)+O\left(N^{5}\right) .
$$

Proof. Consider an edge $e=(u, v)$. Because all edges in a torus are symmetric, without loss of generality, we assume that $v=u+(0,1)$. First we assign weights to edges. Let edge $e_{i}=(u+(i, 0), u+(i, 1)),-(N-1) / 2 \leq i \leq(N-1) / 2$. Define the weight of $e_{i}$ to be $1 /(|i|+1)$. Note that $e=e_{0}$. Let edge $r_{i}=(u+(0,-i), u+(1,-i))$, $0 \leq i \leq(N-1) / 2$. Let edge $r_{i}=(u+(-1,-i), u+(0,-i)),-(N-1) / 2 \leq i \leq-1$. Define the weight of $r_{i}$ to be $1 /(|i|+1)$. Symmetrically we can define the $l_{i}$ 's and their weights. Let edge $l_{i}=(u+(0,-i), u+(-1,-i)), 0 \leq i \leq(N-1) / 2$. Let $l_{i}=(u+(1,-i), u+(0,-i)),-(N-1) / 2 \leq i \leq-1$. Define the weight of $l_{i}$ to be $1 /(|i|+1)$. We define the weights of all other edges to be zero. Figure 14 shows the edges of nonzero weights in a $7 \times 7$ torus.

Now consider a packet $\delta=(s, t)$. Assume that $e$ is in $S E_{\delta}\left(k_{\delta}\right)$ for some $k_{\delta}$. If no shortest path of $\delta$ crosses $e$, then $\delta$ can be omitted in the following. Note that $u=S V_{\delta}\left(k_{\delta}\right)$. Without loss of generality, assume that $\delta$ is a left packet to $e$. So the path that $\delta$ takes contains edges in the $+x$ and $+y$ directions. Let the distance vector from $s$ to $u$ be $\left(x_{1}, y_{1}\right)$, and let the distance vector from $u$ to $t$ be $\left(x_{2}, y_{2}\right)$. Note that $\left|S V_{\delta}\left(k_{\delta}\right)\right|=$ $\min \left(y_{1}, x_{2}\right)+\min \left(x_{1}, y_{2}\right)+1$. Thus

$$
\begin{aligned}
& \left|S V_{\delta}\left(k_{\delta}\right)\right| \geq \min \left(y_{1}, x_{2}\right)+1, \\
& \left|S V_{\delta}\left(k_{\delta}\right)\right| \geq \min \left(x_{1}, y_{2}\right)+1 .
\end{aligned}
$$




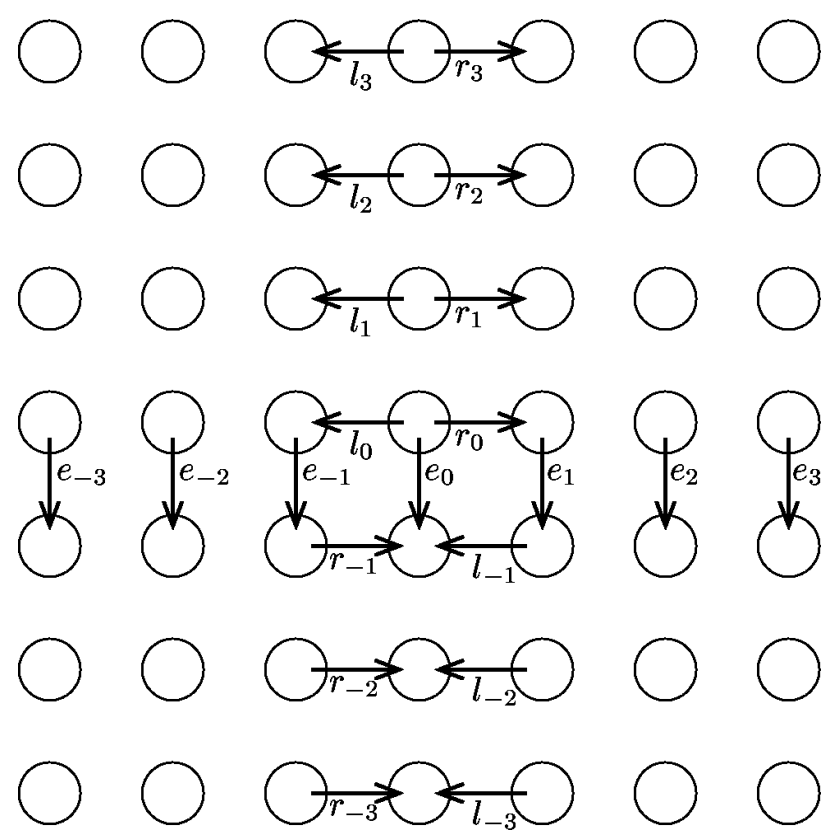

Fig. 14. Edges of nonzero weights in a $7 \times 7$ torus.

Depending on the path $\delta$ takes, one of the following three conditions is true. See Figure 15.

C1. The path contains $e=e_{0}$.

C2. The path contains $r_{i}$ and $e_{j}$, for some $0 \leq i \leq y_{1}$ and $1 \leq j \leq x_{2}$.

C3. The path contains $e_{-i}$ and $r_{-j}$, for some $1 \leq i \leq x_{1}$ and $1 \leq j \leq y_{2}$.

In $\mathrm{C} 1, \delta$ crosses an edge whose weight is 1 . In $\mathrm{C} 2, \delta$ crosses an edge whose weight is at least $1 /\left(\min \left(y_{1}, x_{2}\right)+1\right)$. In $\mathrm{C} 3, \delta$ crosses an edge whose weight is at least $1 /\left(\min \left(x_{1}, y_{2}\right)+1\right)$. By (44) and (45), we conclude:

OBSERVATION 2. $\delta$ must cross an edge whose weight is at least $1 /\left|S V_{\delta}\left(k_{\delta}\right)\right|$.

Let $I_{\delta}$ be the set of packets $\{\varepsilon \mid \varepsilon \in I$ and $\varepsilon$ is equivalent to $\delta\}$. We bound the load on $e$ as follows:

$$
\begin{aligned}
\operatorname{load}_{\mathrm{COUNTER}}(I, e) & \\
& \leq \operatorname{load}_{\mathrm{COUNTER}}(I, u) \\
& =\sum_{\text {nonequivalent } \delta \in I} \operatorname{load}_{\mathrm{COUNTER}}\left(I_{\delta}, u\right) \\
& \leq \sum_{\text {nonequivalent } \delta \in I}\left(\frac{\left|I_{\delta}\right|}{\left|S V_{\delta}\left(k_{\delta}\right)\right|}+N\right) \quad(\text { by Lemma } 4)
\end{aligned}
$$




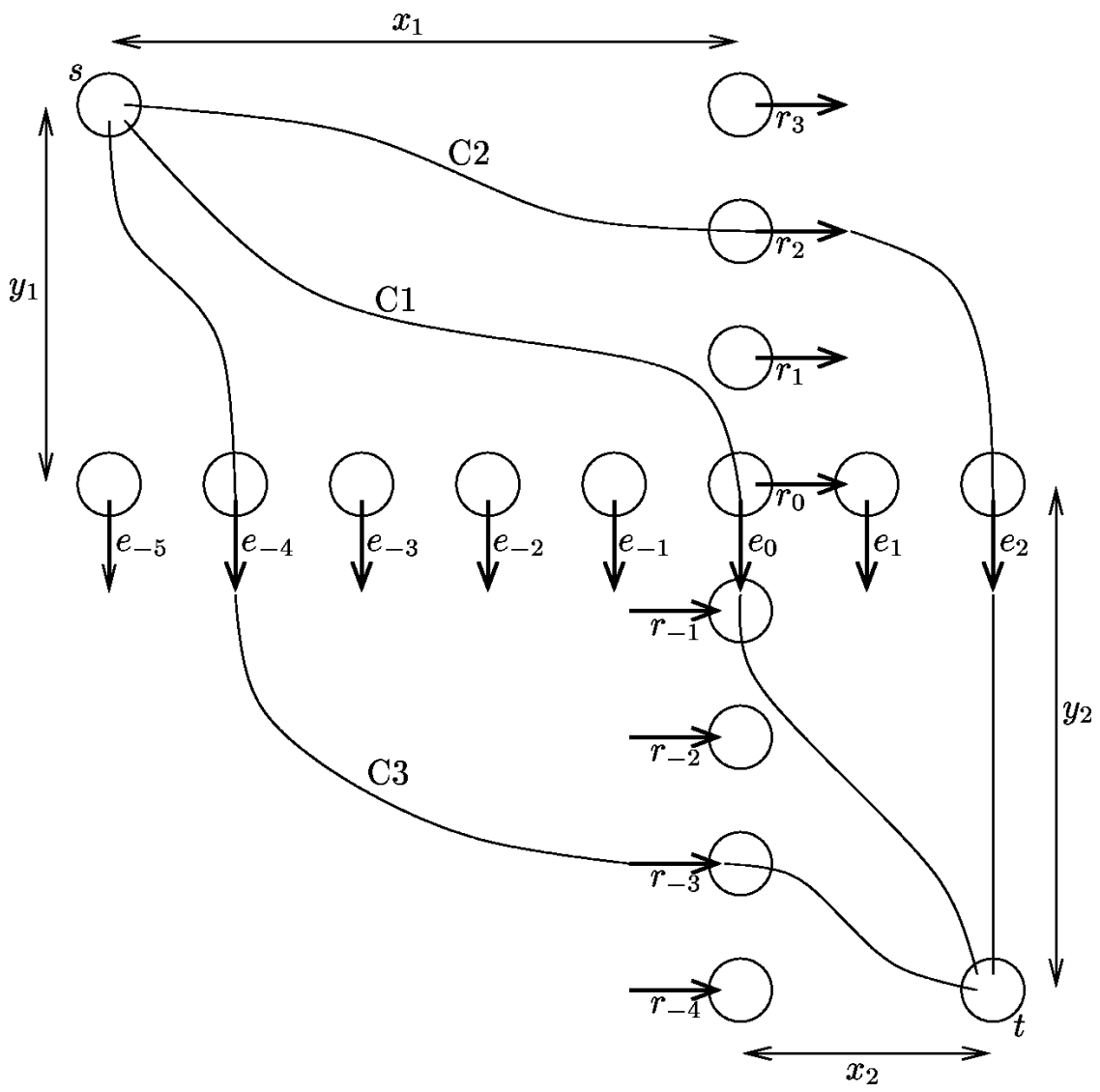

Fig. 15. Paths $\delta$ can take.

$\left.\leq \sum_{\text {nonequivalent } \delta \in I} \frac{\left|I_{\delta}\right|}{\left|S V_{\delta}\left(k_{\delta}\right)\right|}\right)+O\left(N^{5}\right) \quad$ (there are $\leq N^{2} \cdot N^{2}$ nonequivalent $\delta$ 's)

$=\sum_{\delta \in I} \frac{1}{\left|S V_{\delta}\left(k_{\delta}\right)\right|}+O\left(N^{5}\right)$

$\leq \sum_{\delta \in I}$ (weight caused by ADV on $\left.\delta\right)+O\left(N^{5}\right) \quad$ (by Observation 2)

$=\sum_{f \in E}\left((\right.$ weight of edge $\left.f) \times \operatorname{load}_{\mathrm{ADV}}(I, f)\right)+O\left(N^{5}\right)$

$\leq \sum_{f \in E}\left((\right.$ weight of edge $\left.f) \times \operatorname{cost}_{\mathrm{ADV}}(I)\right)+O\left(N^{5}\right)$

$=\left(3 \times 1+6 \times \frac{1}{2}+6 \times \frac{1}{3}+\cdots+6 \times \frac{1}{\lfloor N / 2\rfloor}\right) \times \operatorname{cost}_{\mathrm{ADV}}(I)+O\left(N^{5}\right)$

$=O(\log N) \times \operatorname{cost}_{\mathrm{ADV}}(I)+O\left(N^{5}\right)$. 
This bound is true for arbitrary $e$, so

$$
\operatorname{cost}_{\text {COUNTER }}(I) \leq O(\log N) \times \operatorname{cost}_{\text {ADV }}(I)+O\left(N^{5}\right) .
$$

Note that for a given torus, $N$ is a constant and the additive term $O\left(N^{5}\right)$ is independent of $I$. Therefore, COUNTER is $O(\log N)$ competitive.

5. A Randomized Algorithm. In this section we give a randomized algorithm called "Randomized Counter," abbreviated as RandC. Although we cannot improve on the $O(\log N)$ bound achieved by the deterministic algorithm presented in Section 4 (because the lower bound for the randomized algorithm is $\Omega(\log N)$, as presented in Section 3), the randomized algorithm RandC in this section has the benefit that it is memoryless. By randomness, RandC eliminates the space requirement of the deterministic algorithm.

The intuition behind RandC is similar to that of the deterministic algorithm COUNTER introduced in the previous section. Consider a packet $\delta$ and a slice of nodes $S V_{\delta}(i)$ for some $i$. We design RandC in such a way that the probabilities that $\delta$ visit nodes in $S V_{\delta}(i)$ are uniform. We use the following strategy to achieve this.

Consider an input $I$. RandC processes each packet in $I$ independently. Note that in this way RandC is on-line. Now concentrate on a packet $\delta=(s, t)$. RandC reserves a path for $\delta$ hop by hop. Assume that, after some hops, we are on the node $\langle i, j\rangle$, node $j$ in slice $i$. RandC chooses a node in $S V_{\delta}(i+1)$ for the next hop according to the rules in the following three cases:

Case 1: $\left|S V_{\delta}(i+1)\right|=\left|S V_{\delta}(i)\right|+1$. In this case there are two out-going edges leaving $\langle i, j\rangle$ toward $t$. They go to $\langle i+1, j\rangle$ and $\langle i+1, j+1\rangle$, respectively. RandC chooses $\langle i+1, j\rangle$ as the next node in the path with probability $\left(\left|S V_{\delta}(i)\right|+1-j\right) /\left(\left|S V_{\delta}(i)\right|+1\right)$. RandC chooses $\langle i+1, j+1\rangle$ with probability $j /\left(\left|S V_{\delta}(i)\right|+1\right)$.

Case 2: $\left|S V_{\delta}(i+1)\right|=\left|S V_{\delta}(i)\right|$. In this case RandC chooses the node $\langle i+1, j\rangle$ with probability 1.

Case 3: $\left|S V_{\delta}(i+1)\right|=\left|S V_{\delta}(i)\right|-1$. In this case RandC chooses $\langle i+1, j-1\rangle$ (if it exists) with probability $(j-1) /\left(\left|S V_{\delta}(i)\right|-1\right)$. RandC chooses $\langle i+1, j\rangle$ (if it exists) with probability $\left(\left|S V_{\delta}(i)\right|-j\right) /\left(\left|S V_{\delta}(i)\right|-1\right)$. Note that in this case $\langle i, 1\rangle$ chooses $\langle i+1,1\rangle$ with probability 1 . Similarly, $\left\langle i,\left|S V_{\delta}(i)\right|\right\rangle$ chooses $\left\langle i+1,\left|S V_{\delta}(i+1)\right|\right\rangle$ with probability 1 .

This path selection procedure is done on the source node $s$. So RandC is a source routing algorithm.

LEMMA 5. The probability that $\delta$ visits $\langle i, j\rangle$ is $1 /\left|S V_{\delta}(i)\right|$.

Proof. By induction on $i$. The lemma is true when $i=1$. Assume that the lemma is true in slice $i$, we show that it is true on $\langle i+1, j\rangle$ for some $j$ by considering the following three cases:

Case 1: $\left|S V_{\delta}(i+1)\right|=\left|S V_{\delta}(i)\right|+1$. In this case, only $\langle i, j-1\rangle$ and $\langle i, j\rangle$ have nonzero probability to choose $\langle i+1, j\rangle$ in the next hop. By the induction hypothesis, 
the probabilities that $\delta$ visits $\langle i, j-1\rangle$ or $\langle i, j\rangle$ are both $1 /\left|S V_{\delta}(i)\right|$. The probability to go to $\langle i+1, j\rangle$ from $\langle i, j-1\rangle$ is $(j-1) /\left(\left|S V_{\delta}(i)\right|+1\right)$. The probability to go to $\langle i+1, j\rangle$ from $\langle i, j\rangle$ is $\left(\left|S V_{\delta}(i)\right|+1-j\right) /\left(\left|S V_{\delta}(i)\right|+1\right)$. As a result, the probability of visiting $\langle i+1, j\rangle$ is

$$
\frac{1}{\left|S V_{\delta}(i)\right|} \cdot\left(\frac{j-1}{\left|S V_{\delta}(i)\right|+1}+\frac{\left|S V_{\delta}(i)\right|+1-j}{\left|S V_{\delta}(i)\right|+1}\right)=\frac{1}{\left|S V_{\delta}(i)\right|+1}=\frac{1}{\left|S V_{\delta}(i+1)\right|} .
$$

Case 2: $\left|S V_{\delta}(i+1)\right|=\left|S V_{\delta}(i)\right|$. In this case, only $\langle i, j\rangle$ has nonzero probability to $\langle i+1, j\rangle$. By the induction hypothesis, the probability of visiting $\langle i, j\rangle$ is $1 /\left|S V_{\delta}(i)\right|$. The probability that $\langle i, j\rangle$ extends to $\langle i+1, j\rangle$ is 1 . As a result, the probability of visiting $\langle i+1, j\rangle$ is

$$
1 \cdot \frac{1}{\left|S V_{\delta}(i)\right|}=\frac{1}{\left|S V_{\delta}(i+1)\right|} .
$$

Case 3: $\left|S V_{\delta}(i+1)\right|=\left|S V_{\delta}(i)\right|-1$. In this case, only $\langle i, j\rangle$ (if it exists) and $\langle i, j+1\rangle$ have nonzero probability to $\langle i+1, j\rangle$. The probability to go to $\langle i+1, j\rangle$ from $\langle i, j\rangle$ is $\left(\left|S V_{\delta}(i)\right|-j\right) /\left(\left|S V_{\delta}(i)\right|-1\right)$, and the probability to go to $\langle i+1, j\rangle$ from $\langle i, j+1\rangle$ is $j /\left(\left|S V_{\delta}(i)\right|-1\right)$. As a result, the probability of visiting $\langle i+1, j\rangle$ is

$$
\frac{1}{\left|S V_{\delta}(i)\right|} \cdot\left(\frac{\left|S V_{\delta}(i)\right|-j}{\left|S V_{\delta}(i)\right|-1}+\frac{j}{\left|S V_{\delta}(i)\right|-1}\right)=\frac{1}{\left|S V_{\delta}(i)\right|-1}=\frac{1}{\left|S V_{\delta}(i+1)\right|}
$$

In all three cases the probability that $\delta$ visits $\langle i+1, j\rangle$ is $1 /\left|S V_{\delta}(i+1)\right|$. So the induction is complete. As a result, the lemma follows.

Lemma 5 parallels Lemma 4 for the deterministic algorithm COUNTER. As a result, we have the following lemma for RandC.

LEMMA 6. Consider an input I and an edge e,

$$
\exp \left(\operatorname{load}_{\mathrm{RandC}}(I, e)\right) \leq \omega \log N \operatorname{cost}_{\mathrm{ADV}}(I),
$$

for some constant $\omega$.

PROOF. The derivation of $\exp \left(\operatorname{load}_{\mathrm{RandC}}(I, e)\right)$ is the same as that of $\operatorname{load}_{\mathrm{COUNTER}}(I, e)$ in Theorem 2, except that the $O\left(N^{5}\right)$ additive term is missing here. This is because the additive term $N$ in Lemma 4 is missing in Lemma 5.

In the following we bound the congestion of RandC. We use Chernoff's bound [10] to achieve this. We restate the bound here for easy reference: Let $X_{i}, 1 \leq i \leq m$, be independent 0-1 random variables that $\operatorname{prob}\left(X_{i}=1\right)=p_{i}$. Let $S=\sum_{i=1}^{m} X_{i}$. Note that $\exp (S)=\sum_{i=1}^{m} p_{i}$. Chernoff's bound says that, for any $r \geq 6 \exp (S), \operatorname{prob}(S \geq r) \leq$ $2^{-r}$.

Let $I=\delta_{1} \delta_{2} \cdots \delta_{m}$ be an input of length $m$. Let $X\left(\delta_{i}, e\right)$ be a 0-1 random variable such that $X\left(\delta_{i}, e\right)=1$ if $\delta_{i}$ crosses $e$ and $X\left(\delta_{i}, e\right)=0$ otherwise. In this notation the load on $e$ is $\sum_{i=1}^{m} X\left(\delta_{i}, e\right)$. For convenience, let $S_{e}=\sum_{i=1}^{m} X\left(\delta_{i}, e\right)$. Note that, for a 
fixed edge $e, X\left(\delta_{i}, e\right)$ 's, $1 \leq i \leq m$, are independent 0-1 random variables, because the packets in $I$ are processed independently. (However, $S_{e}$ 's are not independent random variables.) By Lemma $6, \exp \left(S_{e}\right) \leq \omega \log N \operatorname{cost}_{\mathrm{ADV}}(I)$. Let $R=6 \omega \log N \operatorname{cost}_{\mathrm{ADV}}(I)$. By Chernoff's bound, $\operatorname{prob}\left(S_{e} \geq R\right) \leq 2^{-R}$.

Let $C=\max _{e \in E} S_{e}$ be the congestion of the network incurred by RandC. It is easy to see that

$$
\begin{aligned}
\operatorname{prob}(C \geq R) & \leq \sum_{e \in E} \operatorname{prob}\left(S_{e} \geq R\right) \\
& =|E| \cdot 2^{-R} .
\end{aligned}
$$

An upper bound for $\exp (C)$ can be derived as follows (note that $m$ is an upper bound of the congestion $C$ ):

$$
\exp (C) \leq R \cdot \operatorname{prob}(C<R)+m \cdot \operatorname{prob}(C \geq R) \leq R+m \cdot \operatorname{prob}(C \geq R) .
$$

Note that $\operatorname{cost}_{\mathrm{ADV}}(I)$ is at least $m /|E|$. Therefore $R=6 \omega \log N \operatorname{cost}_{\mathrm{ADV}}(I) \geq 6 m \omega$ $\log N /|E|$ and

$$
m \cdot \operatorname{prob}(C \geq R) \leq \frac{m \cdot|E|}{2^{6 m \omega \log N /|E|}} .
$$

Assume that $m=x \cdot 1 /(6 \omega \ln 2) \cdot|E|$ for some $x$. The previous inequality becomes

$$
m \cdot \operatorname{prob}(C \geq R) \leq \frac{(x /(6 \omega \ln 2))\left(4 N^{2}\right)^{2}}{N^{x / \ln 2}} .
$$

Let

$$
f(x)=\frac{(x /(6 \omega \ln 2))\left(4 N^{2}\right)^{2}}{N^{x / \ln 2}}=\frac{8 N^{4}}{3 \omega \ln 2} \cdot \frac{x}{N^{x / \ln 2}} .
$$

The derivative of $f(x)$ is

$$
\frac{d}{d x} f(x)=\frac{8 N^{4}}{3 \omega \ln 2 \cdot N^{x / \ln 2}} \cdot\left(1-\frac{\ln N}{\ln 2} \cdot x\right) .
$$

Notice that when $N \geq 2$ and $x \geq 1$, the derivative of $f(x)$ is negative. This implies that $f(x)$ is a decreasing function when $x \geq 1$. Now choose $x \geq 4 \ln 2 \geq 1$ and correspondingly $m \geq(2 / 3 \omega) \cdot|E|=(8 / 3 \omega) N^{2}$,

$$
m \cdot \operatorname{prob}(C \geq R) \leq\left. f(x)\right|_{x=4 \ln 2}=\frac{32}{3 \omega} .
$$

Therefore, when $m \geq(8 / 3 \omega) N^{2}$,

$$
\exp \left(\operatorname{cost}_{\text {RandC }}(I)\right)=\exp (C) \leq R+m \cdot \operatorname{prob}(C \geq R) \leq R+\frac{32}{3 \omega} .
$$

When $m \leq(8 / 3 \omega) N^{2}$, it is easy to see that

$$
\exp \left(\operatorname{cost}_{\text {RandC }}(I)\right) \leq|I|=m \leq \frac{8}{3 \omega} N^{2} .
$$


Combining the previous two equations, we have

$$
\begin{aligned}
\exp \left(\operatorname{cost}_{\text {RandC }}(I)\right) & \leq R+\max \left(\frac{32}{3 \omega}, \frac{8}{3 \omega} N^{2}\right) \\
& =R+O\left(N^{2}\right)=6 \omega \log N \cdot \operatorname{cost}_{\mathrm{ADV}}(I)+O\left(N^{2}\right) .
\end{aligned}
$$

Because $N$ is a constant for a given torus, the additive term $O\left(N^{2}\right)$ is independent of the input. We conclude this section by the following theorem.

THEOREM 3. The competitive ratio of RandC is $O(\log N)$.

Acknowledgment. We thank the anonymous referees for their helpful comments.

\section{References}

[1] B. Awerbuch and Y. Azar. Local optimization of global objectives: competitive distributed deadlock resolution and resource allocation. In Proceedings of the 35th Annual Symposium on Foundations of Computer Science, pages 240-249, 1994.

[2] B. Awerbuch, Y. Bartal, and A. Fiat. Competitive distributed file allocation. In Proceedings of the Twenty-Fifth Annual ACM Symposium on the Theory of Computing, pages 164-173, 1993.

[3] B. Awerbuch, S. Kutten, and D. Peleg. Competitive distributed job scheduling. In Proceedings of the Twenty-Fourth Annual ACM Symposium on the Theory of Computing, pages 571-580, 1992.

[4] H. Badr and S. Podar. An optimal shortest-path routing policy for network computers with regular mesh-connected topologies. IEEE Transactions on Computers, 38(10):1362-1371, 1989.

[5] Y. Bartal, A. Fiat, and S. Leonardi. Lower bounds for on-line graph problems with applications to circuit and optical routing. In Proceedings of the Twenty-Eighth Annual ACM Symposium on the Theory of Computing, pages 531-540, 1996.

[6] Y. Bartal, A. Fiat, and Y. Rabani. Competitive algorithms for distributed data management. Journal of Computer and System Sciences, 51(3):341-358, 1995.

[7] Y. Bartal and S. Leonardi. On-line routing in all-optical networks. Theoretical Computer Science, 221:19-39, 1999.

[8] S. Ben-David, A. Borodin, R. Karp, G. Tardos, and A. Wigderson. On the power of randomization in on-line algorithms. Algorithmica, 11(1):2-14, 1994.

[9] R. Cypher, F. Meyer auf der Heide, C. Scheideler, and B. Vöcking. Universal algorithms for store-andforward and wormhole routing. In Proceedings of the Twenty-Eighth Annual ACM Symposium on the Theory of Computing, pages 356-365, 1996.

[10] T. Hagerup and C. Rub. A guided tour of Chernoff bounds. Information Processing Letters, 33:305-308, 1990.

[11] F. Leighton, B. Maggs, A. Ranade, and S. Rao. Randomized routing and sorting on fixed-connection networks. Journal of Algorithms, 17(1):157-205, 1994.

[12] F. Leighton, B. Maggs, and S. Rao. Packet routing and job-shop scheduling in $O$ (congestion + dilation) steps. Combinatorica, 14(2):167-186, 1994.

[13] F. Leighton, B. Maggs, and A. Richa. Fast algorithms for finding $O$ (congestion + dilation) packet routing schedules. Combinatorica, 19(2):1-27, 1999.

[14] T. Leighton. Methods for message routing in parallel machines. In Proceedings of the Twenty-Fourth Annual ACM Symposium on the Theory of Computing, pages 77-96, 1992.

[15] B. Maggs, F. Meyer auf der Heide, B. Vöcking, and M. Westermann. Exploring locality for data management in systems of limited bandwidth. In Proceedings of the 38 th Annual Symposium on Foundations of Computer Science, pages 284-293, 1997. 
[16] F. Meyer auf der Heide and B. Vöcking. A packet routing protocol for arbitrary networks. In Proceedings of the 12th Annual Symposium on Theoretical Aspects of Computer Science, pages 291-302, 1995.

[17] R. Ostrovsky and Y. Rabani. Universal $O$ (congestion + dilation $+\log ^{1+\varepsilon} n$ ) local control packet switching algorithms. In Proceedings of the Twenty-Ninth Annual ACM Symposium on Theory of Computing, pages 644-653, 1997.

[18] S. Plotkin. Competitive routing of virtual circuits in ATM networks. IEEE Journal of Selected Areas in Communications, 13(6):1128-1136, 1995.

[19] D. Sleator and R. Tarjan. Amortized efficiency of list update and paging rules. Communications of the ACM, 28:202-208, 1985.

[20] A. Srinivasan and C. Teo. A constant-factor approximation algorithm for packet routing, and balancing local vs. global criteria. In Proceedings of the Twenty-Ninth Annual ACM Symposium on Theory of Computing, pages 636-643, 1997. 